Atmos. Chem. Phys., 10, 475-497, 2010

www.atmos-chem-phys.net/10/475/2010/

(C) Author(s) 2010. This work is distributed under

the Creative Commons Attribution 3.0 License.

\title{
Advective mixing in a nondivergent barotropic hurricane model
}

\author{
B. Rutherford ${ }^{1}$, G. Dangelmayr ${ }^{1}$, J. Persing ${ }^{1}$, W. H. Schubert ${ }^{2}$, and M. T. Montgomery ${ }^{3}$ \\ ${ }^{1}$ Department of Mathematics, Colorado State University, Fort Collins, CO 80523-1874, USA \\ ${ }^{2}$ Department of Atmospheric Science, Colorado State University, Fort Collins, CO 80523-1371, USA \\ ${ }^{3}$ Department of Meteorology, Naval Postgraduate School, Monterey, CA 93943-5114, USA
}

Received: 18 June 2009 - Published in Atmos. Chem. Phys. Discuss.: 28 July 2009

Revised: 29 November 2009 - Accepted: 29 December 2009 - Published: 20 January 2010

\begin{abstract}
This paper studies Lagrangian mixing in a twodimensional barotropic model for hurricane-like vortices. Since such flows show high shearing in the radial direction, particle separation across shear-lines is diagnosed through a Lagrangian field, referred to as $R$-field, that measures trajectory separation orthogonal to the Lagrangian velocity. The shear-lines are identified with the level-contours of another Lagrangian field, referred to as $S$-field, that measures the average shear-strength along a trajectory. Other fields used for model diagnostics are the Lagrangian field of finite-time Lyapunov exponents ( $F T L E$-field), the Eulerian $Q$-field, and the angular velocity field. Because of the high shearing, the FTLE-field is not a suitable indicator for advective mixing, and in particular does not exhibit ridges marking the location of finite-time stable and unstable manifolds. The FTLE-field is similar in structure to the radial derivative of the angular velocity. In contrast, persisting ridges and valleys can be clearly recognized in the $R$-field, and their propagation speed indicates that transport across shear-lines is caused by Rossby waves. A radial mixing rate derived from the $R$ field gives a time-dependent measure of flux across the shearlines. On the other hand, a measured mixing rate across the shear-lines, which counts trajectory crossings, confirms the results from the $R$-field mixing rate, and shows high mixing in the eyewall region after the formation of a polygonal eyewall, which continues until the vortex breaks down. The location of the $R$-field ridges elucidates the role of radial mixing for the interaction and breakdown of the mesovortices shown by the model.
\end{abstract}

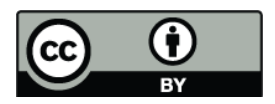

Correspondence to: B. Rutherford (rutherfo@math.colostate.edu)

\section{Introduction}

Several recent studies (Frank and Ritchie, 1999, 2001; Montgomery et al., 2006; Hendricks and Schubert, 2009) are devoted to the mixing of fluid from different regions of a hurricane, which is considered as a fundamental mechanism that is intimately related to hurricane intensity. A complete understanding of these mixing processes, in particular the eyeeyewall mixing (Bryan and Rotunno 2009a, b; Cram et al., 2007; Braun et al., 2006; Montgomery et al., 2002, 2006; Willoughby, 2001), is expected to improve our understanding of the physical mechanisms that regulate hurricane intensity. Since mixing is based on particle motion, the Lagrangian frame of reference provides the most natural framework in which it can be diagnosed. Much progress has been made in recent years in the study of Lagrangian mixing in twodimensional incompressible flows (Haller and Poje, 1997; Haller and Yuan, 2000; Haller, 2000, 2001, 2002; Shadden et al., 2005), resulting in a number of different, though related diagnostics, most of which are based on concepts from dynamical systems theory. For applications of Lagrangian techniques to atmospheric models, see Joseph and Legras (2001) and Huber et al. (2001).

Much insight into specific aspects of mixing in hurricanes can be gained from the study of simplified two-dimensional models. Basically there are two classes of such models: Axisymmetric models, most notably the model of Rotunno and Emanuel (1987), and planar models such as the model of Kossin and Schubert (2001) and Schubert et al. (1999). In this paper we apply Lagrangian techniques to analyze mixing in the planar, nondivergent barotropic model of Kossin and Schubert (2001). Our analysis confirms a study of Kossin and Eastin (2001) which illustrates that significant

Published by Copernicus Publications on behalf of the European Geosciences Union. 
eye-eyewall mixing occurs during polygonal eyewall transitions. Lagrangian mixing in the axisymmetric model introduced in Rotunno and Emanuel (1987) is investigated in Rutherford et al. (2009). For another discussion of axisymmetric mixing, see Wirth and Dunkerton (2006).

The model studied in this paper provides a twodimensional representation of a hurricane that initiates with an annular ring of enhanced vorticity, and then undergoes a vortex breakdown resulting in a monopolar end state. During the breakdown, a polygonal eyewall occurs, which forms four elliptical pools of high vorticity. Mixing of potential vorticity, which in this model is proportional to relative vorticity, can be visualized using Eulerian diagnostic measures of instantaneous particle separation. A commonly used Eulerian diagnostic is the so called $Q$-field, derived from the Jacobian of the Eulerian velocity field. According to the Okubo-Weiss criterion (Schubert et al., 1999), positive values of this field indicate instantaneous particle separation, whereas negative values indicate contraction. For our model, the $Q$-field shows that regions of high relative vorticity gradient are also places where high trajectory separation and mixing occurs.

While Eulerian measures of mixing can only diagnose instantaneous particle separation, Lagrangian techniques utilize a moving frame approach along trajectories and compute measures for the average separation over a finite integration time. This approach is particularly useful in timedependent velocity fields, where trajectories may cross Eulerian streamlines (Dunkerton et al., 2009). Much of the recent work in Lagrangian mixing has extended the ideas of hyperbolicity for steady flows to time dependent velocity fields (Haller, 2002; Haller and Poje, 1997; Haller and Yuan, 2000; Green et al., 2006; Ide et al., 2002), generalizing the concept of stable and unstable manifolds of an equilibrium to the stable and unstable manifolds of a hyperbolic trajectory. These manifolds are referred to as Lagrangian coherent structures (LCS's). Even in two-dimensional flows, timedependence can give rise to multiple intersections of these manifolds, leading to a partition of the flow into invariant regions (lobes), and to mixing through the lobe dynamics (Ide et al., 2002).

Efficient visualization of LCS's is accomplished through the construction of Lagrangian scalar fields, which measure separation of nearby trajectories. Current Lagrangian methods utilize a variety of fields, including finite-time Lyapunov exponents (Haller, 2002; Haller and Poje, 1997; Haller and Yuan, 2000), finite-size Lyapunov exponents (Koh and Legras, 2002; Green et al., 2006), and relative dispersion (Huber et al., 2001). Each of these methods defines a scalar field and the LCS's as maximal ridges of that field.

To study Lagrangian mixing in our model, we compute particle trajectories from the numerically calculated, timevarying velocity field. The Lagrangian diagnostic fields are functions of the initial time and position at which the trajectories are seeded. A comparison of these fields with the
Okubo-Weiss criterion indicates that high particle separation predicted from the $Q$-field typically does not coincide with Lagrangian hyperbolic structures, however the Lagrangian $Q$-field, formed by integrating $Q$-values along particle trajectories, shows a greater relation to other Lagrangian fields.

An important feature of the particle trajectories calculated from our model is that they show an almost circular motion, combined with high shearing in the radial direction. The problem caused by this high shear is that trajectory separation and mixing occur without the entrainment of trajectories, as the mixing is largely diffusive. A key question that we aim to answer is whether coherent structures that play a role in the systematic transport of trajectories can persist through high shear.

Distinct regions of trajectories with similar properties become more difficult to distinguish through the use of scalar fields which measure only distance, such as the field of finitetime Lyapunov exponents (FTLE-field). In fact, the FTLEfield computed from our model does not show distinguished ridges characteristic of hyperbolic mixing. Instead, the structure of the FTLE-field is very similar to the structure of the radial derivative of the angular velocity, indicating that the FTLE-field is dominated by the shear and not by hyperbolic mixing.

In order to separate shear from hyperbolic mixing we follow the approach used in Haller and Iacono (2003), and decompose the separation of trajectories in the directions along and normal to the Lagrangian velocity. This approach allows us to identify two Lagrangian fields: The $R$-field, which is a diagnostic for hyperbolic mixing normal to the Lagrangian velocity, and the $S$-field, which is a measure of shearing, and is used to define shear-lines by its level-contours. In contrast to the FTLE-field, the $R$-field shows distinct ridges and valleys observable as coherent structures. The evolution of these structures provides a mechanism for mixing through the eyewall, and their speed indicates that this mixing is caused by Rossby waves. The structures are particularly distinct after polygonal eyewall formation, and they persist until the vortex breaks down, in regions where the Okubo-Weiss criterion predicts pools of high separation associated with the formation of pools of high vorticity.

We note that another approach to diagnosing mixing in the presence of shear is based on subtracting a mean shear from the flow. This approach was introduced by Andrews and McIntyre (1978) using a generalized Lagrangian mean for nonlinear waves, and was subsequently developed further and refined to a modified Lagrangian mean to quantify and distinguish stirring from irreversible mixing, see McIntyre (1980) and Dunkerton (1980).

The time-dependence of our velocity field leads to timedependent shear-lines, and regions of high orthogonal (hyperbolic) separation lead to sets of trajectories that are mixed through the shear-lines. We quantify this mixing by introducing measured (via trajectory counting) and predicted (from the $R$-field) mixing rates. In addition, we study radial mixing 
rates defined by angular averages of the FTLE-field, the $S$ field, and the $R$-field. The mixing rates defined through the former two fields are characteristic of shearing and give spuriously a false sense of mixing during the initial phase of the model, where "true mixing" occurs after the polygonal eyewall formation.

In previous work on the same model, Hendricks and Schubert (2009) have applied the Lagrangian-Eulerian hybrid method of effective diffusion (Nakamura, 1996; Shuckburgh and Haynes, 2003). Here diffusive mixing properties are computed based on the increasing lengths of the vorticity contours, with the computations initialized at the initial time of the model. The resulting mixing rate is a function of an effective radius and the integration time, and shows similar structures as our mixing rates.

Our methods depart from those of Hendricks and Schubert (2009) in that we utilize a moving time window, which attributes mixing to short-time advective events. Rather than determining contour lengths, we study transport across contours of the $S$-field. The resulting mixing rates are completely determined by the given velocity field, that is, they do not depend on a chosen initial profile of the tracer distribution.

The outline of the paper is as follows. We begin, in Sect. 2, with an overview of the nondivergent barotropic model, and of the numerical methods used to compute the velocity field and the particle trajectories. In Sect. 3 we introduce the scalar fields utilized for diagnosing mixing and shear: The Eulerian $Q$-field, the Lagrangian $Q$-field, the angular velocity field, the Lagrangian FTLE-field, the $R$-field, and the $S$-field. The latter two fields are extracted from the transformed variational system introduced in Haller and Iacono (2003). The main results of the paper are presented in Sects. 4 to 6 . In Sect. 4 we study the behavior of the three Lagrangian fields for a fixed initial time of $6 \mathrm{~h}$, after a polygonal eyewall has formed, and for different integration times. The ridge, valley, and edge structures observed in the $R$-field are identified with coherent structures and invariant sets relative to the shearing. In Sect. 5 we fix the integration time to $1 \mathrm{~h}$ and study the diagnostic fields for varying initial times. The structures observed in these fields are related to different mixing processes occurring during the three main phases of the model: crystalization in which polygonal eyewall features form and develop filamentation, vortex interaction and merger which destroy the symmertry, and final collapse into a monopole. Section 6 is devoted to the mixing rates mentioned before, which are displayed as functions of initial time and either radius or value of $S$ along a shear-line. Concluding remarks and an outlook on future work are given in Sect. 7.

\section{Model overview}

The model used in this paper is the 2-D nondivergent barotropic model for hurricane-like vortices studied by Kossin and Schubert (2001); Kossin and Eastin (2001); Schubert et al. (1999). The velocity field, $\mathbf{u}(\mathbf{x}, t)=(u(\mathbf{x}, t), v(\mathbf{x}, t))^{*}$ with $\mathbf{x}=(x, y)^{*} \in \mathbb{R}^{2}$ (asteriks denote transposed vectors or matrices), is given as the solution on the $f$-plane of the incompressible Navier Stokes equation,

$$
\begin{aligned}
& \frac{\partial \mathbf{u}}{\partial t}+(\mathbf{u} \cdot \nabla) \mathbf{u}-f \mathbf{B u}+\frac{1}{\rho} \nabla p=v \nabla^{2} \mathbf{u}, \\
& \nabla \cdot \mathbf{u}=0,
\end{aligned}
$$

where

$\mathbf{B}=\left(\begin{array}{rr}0 & 1 \\ -1 & 0\end{array}\right)$,

$p$ is the pressure, $\rho$ the constant density, $f$ the constant Coriolis parameter, and $v$ the constant viscosity, chosen to be $100 \mathrm{~m}^{2} \mathrm{~s}^{-1}$. In the choice of $v$ we follow Schubert et al. (1999), while Kossin and Schubert (2001) used $5 \mathrm{~m}^{2} \mathrm{~s}^{-1}$. The choice of viscosity may have an effect on long time mixing processes, which could be studied by the methods of Hendricks and Schubert (2009). Expressing the velocity in terms of a streamfunction $\psi(\mathbf{x}, t)$ as $\mathbf{u}=-\mathbf{B} \nabla \psi$ and eliminating the pressure from Eq. (1), leads to the equation

$\frac{\partial \zeta}{\partial t}+\frac{\partial \psi}{\partial x} \frac{\partial \zeta}{\partial y}-\frac{\partial \psi}{\partial y} \frac{\partial \zeta}{\partial x}=v \nabla^{2} \zeta$,

where $\zeta=\nabla^{2} \psi$ is the relative vorticity. Following Kossin and Schubert (2001), we impose periodic boundary conditions on $\psi$ with a fundamental domain of $600 \mathrm{~km} \times 600 \mathrm{~km}$, and choose as initial condition an almost circular symmetric ring of vorticity, $\zeta_{0}(r, \theta)$, to model a 2-D hurricane after an initial eyewall has formed. The defining equation of $\zeta_{0}(r, \theta)$ is the equation used in Kossin and Schubert (2001).

Equation (3) was solved numerically using a Fourier pseudospectral method with $512 \times 512$ collocation points. Dealiasing results in $170 \times 170$ Fourier modes. The ODEsystem for the Fourier modes was solved via Matlab's ode45 routine, which implements a fourth order Runge-Kutta method with adaptive time steps.

In our numerical calculation of $\psi$ and $\zeta$, we reproduced the behavior observed in Schubert et al. (1999). The annular ring of high vorticity fluid develops a wavenumber 4 asymmetry, which is present in the vorticity fields as early as $2 \mathrm{~h}$, and develops into a polygonal eyewall, with 4 mesovortices after $6 \mathrm{~h}$. After $8 \mathrm{~h}$, the mesovortices begin to break down and merge. The breakdown of the mesovortices is nearly complete after $12 \mathrm{~h}$, and mixing of high and low vorticity occurs along long filament structures. The relative vorticity fields during these times can be seen in Figs. 6 to 11a. After 24h, diffusive mixing along the filaments leads to a more mixed state. Few pools of high or low vorticity fluid remain, with a 
pool of low-vorticity fluid from the eye migrating through the eyewall, and high vorticity fluid redistributing in the eye. After $48 \mathrm{~h}$, a high vorticity eye and a low vorticity environment remain in a monopole endstate. The eyewall is no longer present as there is no longer a strong angular velocity gradient.

In this paper we study Lagrangian mixing in the model, which is based on following trajectories for varying initial times. The trajectories were calculated with the same spatial and temporal resolution as the model output, using a fourth order Runge-Kutta method with a fixed time step of $7.5 \mathrm{~s}$. Because of time and memory limitations associated with the large number of trajectories needed for quantifying mixing over a sequence of initial times, the trajectories used for computing time-dependent mixing rates were calculated with a time step of $60 \mathrm{~s}$. Comparison of the results for the two time steps for a small random set of initial conditions showed that the use of the coarser time resolution in the mixing calculations is justified.

\section{Diagnostic fields for mixing and shear}

In this section we introduce the scalar fields utilized to diagnose the particle flow resulting from the numerically calculated velocity field. A main characteristic feature of the model is an almost circular motion, the trajectories encircle the origin in the counterclockwise direction. The model shows a strong variation of the particle speed $|\mathbf{u}|$ in the radial direction. This variation leads to high shearing that dominates the particle separation, but is not the result of hyperbolic trajectory separation. Superimposed on this shear effect is hyperbolic mixing due to trajectory separation in directions orthogonal to the velocity.

In order to diagnose hyperbolicity, we exploit the Lagrangian field introduced in Haller and Iacono (2003), in which hyperbolic trajectory splitting is separated from particle separation due to shearing. The more common FTLEfield is also analyzed, however, this field is dominated by the shear and hence not suitable as an indicator for hyperbolic mixing. In order to quantify hyperbolic mixing, we define closed shear lines as contour lines of a suitably defined shear field, and measure and predict transport across these lines (Sect. 6). Further indicators used in our study are two Eulerian fields: The Hessean determinant of the streamfunction ( $Q$-field), and the radial gradient of the instantaneous angular velocity.

\subsection{Eulerian fields}

\subsubsection{Q-field}

Eulerian trajectory separation occurs when the linearized velocity shows local expansion of area. The local variation of area can be inferred from the Jacobian of the velocity field, that is, from the Hessian determinant of the streamfunction, which is referred to as the $Q$-field,

$Q(\mathbf{x}, t)=\psi_{x y}^{2}(\mathbf{x}, t)-\psi_{x x}(\mathbf{x}, t) \psi_{y y}(\mathbf{x}, t)$.

According to the Okubo-Weiss criterion (Schubert et al., 1999), regions with $Q>0$ show local trajectory repulsion, whereas regions with $Q<0$ show local attraction. The $Q$ field allows diagnosis of instantaneous separation, which typically differs from Lagrangian measures of separation.

\subsubsection{Angular velocity}

The strong rotation and near symmetry of the flow suggests that polar coordinates $(r, \theta)$ provide a useful coordinate system for displaying fields calculated from the velocity field. In particular, the quasi-circular behavior of trajectories suggests that the angular velocity, $\omega=r^{-1} \mathbf{u} \cdot(-\sin \theta, \cos \theta)^{*}$, is an approximate measure of the particle speed, and the derivative $\partial \omega / \partial r$ is an approximate measure of shearing.

For any scalar field $\varphi(\mathbf{x}, t)$, a measure for the radial variation is provided by the angular average, indicated by an overbar,

$\bar{\varphi}(r, t)=\frac{1}{2 \pi} \int_{0}^{2 \pi} \varphi(r, \theta, t) d \theta$.

Contours of $\frac{\partial \omega}{\partial r}$ are shown in Figs. 6 to $11 \mathrm{~d}-\mathrm{f}$ showing the relationship of maxima (maximum normal propogating shear), and minima (maximum counter propogating shear) of $\frac{\partial \omega}{\partial r}$ to features of other scalar fields.

\subsection{Lagrangian fields}

Let $\phi_{t_{0}}^{t}\left(\mathbf{x}_{0}\right)$ be the flow map associated with the equation

$\dot{\mathbf{x}}=\mathbf{u}(\mathbf{x}, t)$

for particle trajectories $\mathbf{x}(t)$, that is, the solution of Eq. (5) with initial condition $\mathbf{x}\left(t_{0}\right)=\mathbf{x}_{0}$. Small perturbations in the initial condition, $\mathbf{y}_{0}=\mathbf{x}_{0}+\boldsymbol{\xi}_{0}$, lead to a perturbed trajectory $\mathbf{y}(t)=\mathbf{x}(t)+\boldsymbol{\xi}(t)$. For sufficiently small $\left|\boldsymbol{\xi}_{0}\right|$, the perturbation $\xi(t)$ can be approximated through the Jacobian of the flow map as

$\xi(t)=\nabla \phi_{t_{0}}^{t}\left(\mathbf{x}_{0}\right) \boldsymbol{\xi}_{0}$,

which satisfies the variational equation

$\dot{\xi}=\nabla \mathbf{u}(\mathbf{x}(t), t) \boldsymbol{\xi}$.

\subsubsection{Finite time Lyapunov exponents}

Finite time Lyapunov exponents have become a standard indicator for Lagrangian trajectory separation (Haller and Poje, 1997; Haller, 2000; Haller and Yuan, 2000; Haller, 2002). Consider a time range $\left[t_{0}, t_{0}+T\right]$ with fixed integration time 
$T$. The growth of $|\boldsymbol{\xi}(t)|$ during this time range is governed by the Cauchy-Riemann deformation tensor,

$\Delta\left(\mathbf{x}_{0}, t_{0}\right)=\left(d_{\mathbf{x}_{0}} \phi_{t_{0}}^{t_{0}+T}\left(\mathbf{x}_{0}\right)\right)^{*}\left(d_{\mathbf{x}_{0}} \phi_{t_{0}}^{t_{0}+T}\left(\mathbf{x}_{0}\right)\right)$,

and becomes maximal when $\xi_{0}$ is aligned with the eigenvector corresponding to the larger eigenvalue, $\lambda_{\max }(\Delta)$, of $\Delta$. The quantity

$\sigma\left(\mathbf{x}_{0}, t_{0}\right)=\frac{1}{2|T|} \ln \lambda_{\max }\left(\Delta\left(\mathbf{x}_{0}, t_{0}\right)\right)$

is the FTLE-field (considered as a function of $\mathbf{x}_{0}$ ) at the initial time $t_{0}$. One distinguishes forward FTLE-fields $(T>0)$ and backward FTLE-fields $(T<0)$. Maximal ridges of the forward and backward FTLE-fields play the role of unstable (repelling) and stable (attracting) manifolds over the time range considered (Haller, 2000; Shadden et al., 2005). In the limit $T \rightarrow \infty$ or $T \rightarrow-\infty, \sigma$ approaches the true Lyapunov exponent, and the maximal ridges approach the stable and unstable manifolds, of the full trajectory seeded at $\left(\mathbf{x}_{0}, t_{0}\right)$. Data limitations in the case of numerically computed velocity fields allow only finite time integrations, and restrict the analysis to FTLE's.

For our velocity field, the particle separation is dominated by the shearing in the radial direction. As a result, the FTLEvalues measure growth of perturbations in approximately angular directions, and high FTLE-values (ridges) occur near extrema of $\partial \omega / \partial r$, whereas low FTLE-values occur near zero contours of $\partial \omega / \partial r$. Generally, FTLE-fields are not suitable as indicators of hyperbolic mixing in the presence of high shear.

\subsubsection{Integrated $Q$-field}

In addition to the instantaneous $Q$-field, Eq. (4), we consider the integrated $Q$-field, formed by integrating $Q$ along trajectories

$\widehat{Q}(\mathbf{x}, t)=\int_{t_{0}}^{t_{0}+T} Q\left(\mathbf{x}_{\mathbf{0}}(t), t\right) d t$.

\subsubsection{Lagrangian fields for hyperbolic mixing and shear}

Following Haller and Iacono (2003), in order to separate mixing and shear in the variational system (Eq. 7), a moving frame of reference is introduced by setting

$\xi=\mathbf{M}(\mathbf{x}(t), t) \boldsymbol{\eta}$,

where the component vectors of the matrix $\mathbf{M}$,

$$
\mathbf{M}(\mathbf{x}, t)=\frac{1}{|\mathbf{u}(\mathbf{x}, t)|}\left(\mathbf{u}(\mathbf{x}, t), \mathbf{u}^{\perp}(\mathbf{x}, t)\right), \mathbf{u}^{\perp}=(-v, u)^{*},
$$

are the normalized fluid velocity $\mathbf{u} /|\mathbf{u}|$, and the unit vector orthogonal to $\mathbf{u}$. This transformation is motivated by the fact that for autonomous velocity fields, $\mathbf{u}=\mathbf{u}(\mathbf{x}), \mathbf{u}(\mathbf{x}(t))$ is a solution of Eq. (7). Although our numerically computed fluid velocity is non-autonomous, its time variation is slow, so that $\mathbf{u}(\mathbf{x}(t), t)$ is still close to a solution of Eq. (7) for finite times. The transformed system for $\boldsymbol{\eta}$ can be written in the form (Haller and Iacono, 2003),

$\dot{\boldsymbol{\eta}}=[\mathbf{A}(t)+b(t) \mathbf{B}] \boldsymbol{\eta}$,

where

$$
\begin{aligned}
& \mathbf{A}(t)=\left(\begin{array}{cr}
-r(\mathbf{x}(t), t) & a(\mathbf{x}(t), t) \\
0 & r(\mathbf{x}(t), t)
\end{array}\right), \\
& b(t)=\left.\frac{1}{|\mathbf{u}|^{2}} \mathbf{u}^{\perp} \cdot \mathbf{u}\right|_{\mathbf{x}=\mathbf{x}(t)}, \\
& r(\mathbf{x}, t)=\frac{1}{|\mathbf{u}|^{2}}\left(\mathbf{u}^{\perp}\right)^{*}(\nabla \mathbf{u}) \mathbf{u}^{\perp} \\
& =\frac{1}{|\mathbf{u}|^{2}}\left(u^{2} v_{y}-u v\left(u_{y}+v_{x}\right)+v^{2} u_{x}\right),
\end{aligned}
$$

and the non-diagonal entry $a$ is composed of two parts,

$a(\mathbf{x}, t)=s(\mathbf{x}, t)+d(\mathbf{x}, t)$,

where

$$
\begin{aligned}
& s(\mathbf{x}, t)=\frac{1}{|\mathbf{u}|^{2}} \mathbf{u}^{*}(\nabla \mathbf{u}) \mathbf{u}^{\perp} \\
& =\frac{1}{|\mathbf{u}|^{2}}\left(u^{2} u_{y}+u v\left(v_{y}-u_{x}\right)-v^{2} v_{x}\right), \\
& d(\mathbf{x}, t)=\frac{1}{|\mathbf{u}|^{2}}\left(\mathbf{u}^{\perp}\right)^{*}(\nabla \mathbf{u}) \mathbf{u} \\
& =\frac{1}{|\mathbf{u}|^{2}}\left(u^{2} v_{x}+u v\left(v_{y}-u_{x}\right)-v^{2} u_{y}\right) .
\end{aligned}
$$

The terms in the transformed linearized system (Eq. 10) motivate the definition of Lagrangian fields as diagnostics for hyperbolic mixing and shear. Since our velocity field is slowly varying in time, the terms associated with $b(t)$ in Eq. (10) are neglected in these definitions.

\subsection{R-field}

As a consequence of incompressibility, the matrix $\mathbf{A}(t)$ has the eigenvalues $\pm r(\mathbf{x}(t), t)$. Fixing an integration time $T$, the integrated field $R$,

$R\left(\mathbf{x}_{0}, t_{0}\right)=\int_{t_{0}}^{t_{0}+T} r\left(\phi_{t_{0}}^{t_{0}+\tau}\left(\mathbf{x}_{0}\right), \tau\right) d \tau$,

describes the growth of a perturbation in the direction orthogonal to the Lagrangian velocity, and the ratio $R /|T|$ plays the role of a finite-time Lyapunov exponent in this direction. Thus $R$ is a measure of attraction $(R<0)$ or repulsion $(R>0)$ of nearby trajectories towards $\mathbf{x}(t)$ over the integration interval $\left[t_{0}, t_{0}+T\right]$. Due to incompressibility, expansion orthogonal to $\mathbf{u}$ is combined with contraction in the direction of $\mathbf{u}$ and vice versa. 
We note that in the limit $T \rightarrow \infty, R$ reduces to the mixing efficiency proposed by Ottino (1989), when this efficiency is evaluated in the direction orthogonal to $\mathbf{u}$. In our study, $R$ will be used as the main diagnostic field for hyperbolicity, and in addition as a means to predict mixing rates across the shear-lines defined below.

\subsection{S-field}

The term $s(\mathbf{x}, t)$ can be written in the form

$s(\mathbf{x}, t)=(\nabla|\mathbf{u}(\mathbf{x}, t)|) \cdot \mathbf{u}^{\perp}(\mathbf{x}, t)$,

and hence characterizes the rate of change of the particle speed in the direction orthogonal to the velocity. Thus $s$ is a local, Eulerian measure of shear in the fluid flow. We define the $S$-field by integrating $s$ along trajectories,

$S\left(\mathbf{x}_{0}, t_{0}\right)=\int_{t_{0}}^{t_{0}+T} s\left(\phi_{t_{0}}^{t_{0}+\tau}\left(\mathbf{x}_{0}\right), \tau\right) d \tau$,

and use $S$ as a Lagrangian diagnostic field for shearing.

We note that an alternative Lagrangian measure of shear has been defined in Haller and Iacono (2003) using the nondiagonal entry of the fundamental matrix of $\dot{\boldsymbol{\eta}}=\mathbf{A}(t) \boldsymbol{\eta}$. However, this field involves a double time-integral and is computationally more expensive. The $S$-field has a straightforward interpretation as shear-diagnostic due to Eq. (12), and requires less computational effort.

As in the case of the FTLE-field, we distinguish forward $(T>0)$ and backward $(T<0)$ fields for both $R$ and $S$.

\subsection{Shear-lines}

For a given integration time $T$, we define the shear-line of strength $C$ at initial time $t_{0}$ as the level contour of $S$, i.e.,

$S_{C}=\left\{\mathbf{x}_{0} \mid S\left(\mathbf{x}_{0}, t_{0}\right)=C\right\}$

- High values of $|C|$ correspond to lines with high shear. For our model, the shear lines are all closed curves around the origin (distorted circles). Positive and negative values of $S$ indicate that the Lagrangian speed increases when moving radially outwards and inwards, respectively. We refer to the first case as "normal propagating shear" and to the second case as "counter-propagating shear". Hyperbolic mixing measured by $R$ is associated with transport across the shearlines. This will be used in Sect. 6 to define mixing rates.

\section{Lagrangian fields and coherent structures}

In Figs. 1, 2, and 3 we show forward and backward FTLE-, $S$-, and $R$-fields, respectively, at the initial time $t_{0}=6 \mathrm{~h}$, after the polygonal eyewall has formed, and for integration times $T= \pm 15 \mathrm{~min}, T= \pm 30 \mathrm{~min}$, and $T= \pm 120 \mathrm{~min}$. For $T= \pm 15 \mathrm{~min}$, the FTLE-field (Fig. 1a,b) reveals coherent structures near the polygonal eyewall, since the effect of the shear is not so pronounced over this short time range. For increasing $|T|$ the shear becomes dominant, and the FTLElevel contours evolve into distorted circles (Fig. 1c-f). Comparison of the azimuthally averaged fields (Sect. 5) shows that high FTLE-values occur near extreme values of $\overline{\partial \omega / \partial r}$. While high FTLE-values correspond to high trajectory separation, they do not give clear LCS's (ridges) for longer integration times. LCS's can be seen only at very short integration times (Fig. 1a and b), and in regions that are predicted by the $Q$-field. As integration time is increased (Fig. 1cf), the LCS's do lengthen as expected, but they also become broader. In particular, the LCS's for $T= \pm 15$ min that are located near the corners of the eyewall, converge into a single broad ring that represents an annulus of high shear.

The $S$-field (Fig. 2) is a shear-indicator, and its level contours (the shear-lines) are distorted circles for all integration times. The FTLE-field shows similar structures as the $S$-field for longer integration times, confirming that trajectory separation is mainly due to shear.

The $R$-field (Fig. 3) shows structures of high and low $R$ values that persist over a series of integration times, making them coherent. These structures lengthen and become more resolved (narrower) when the integration time increases. Initial points on ridges and valleys have $R>0$ and $R<0$, indicating strong separation and contraction in the (approximately radial) direction orthogonal to the Lagrangian velocity, respectively. The structures exist in both the forward and backward time fields, and some of the forward and backward time structures have intersection points. Since the $R$-field is radially continuous, high values of the $R$-field lead to trajectories that show high net movement orthogonal to the Lagrangian velocity, and hence are more likely to cross shear-lines. Since the structures span across the shear-lines, they are not Lagrangian, as trajectories with high angular velocity pass trajectories with lower angular velocity.

A prominent feature of the forward $R$-field at $t_{0}=6 \mathrm{~h}$ (and later) are the filaments observable in Fig. 3a, c and e, which are a consequence of the polygonal eyewall. At $t_{0}=2 \mathrm{~h}$ (Fig. 4a) no filaments are observed. The filamentation concerns the ridges and valleys, as well as the edges between them.

\subsection{An advective mixing mechanism}

Initial conditions $\mathbf{x}_{0}$ that satisfy $R\left(\mathbf{x}_{0}, t_{0}\right)=0$ are invariant in the sense that there is no net movement of neighboring trajectories relative to the Lagrangian velocity. As can be seen in Figs. 3 and $4 \mathrm{a}$, ridges and valleys of $R$ come in nearby pairs, and the ridge and valley of a pair are separated by a segment of a zero contour which forms (approximately) an edge of the $R$-field. The edge is neutrally stable, that is, it attracts from one side (from the ridge) and repels from the other side (towards the valley). The situation is illustrated in Fig. $4 \mathrm{a}, \mathrm{c}$ and $\mathrm{d}$ for $t_{0}=2 \mathrm{~h}$. At this initial time the motion is 


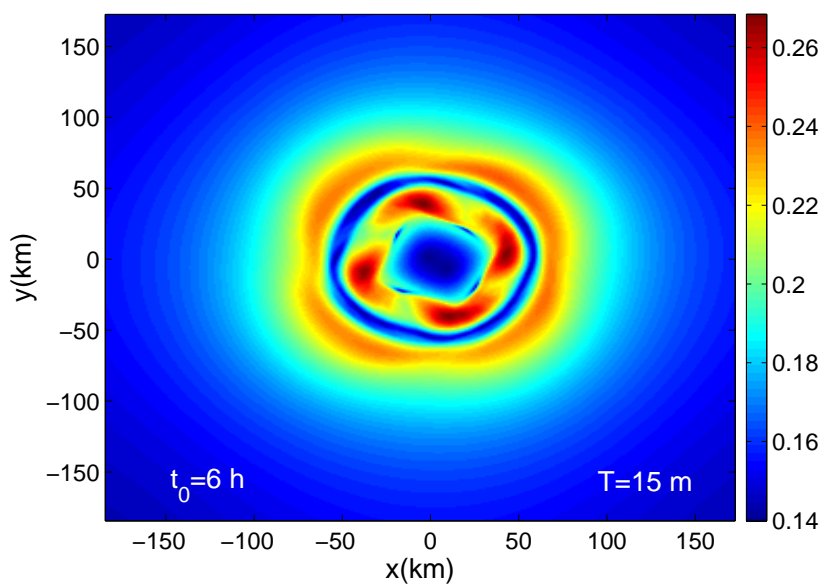

(a)

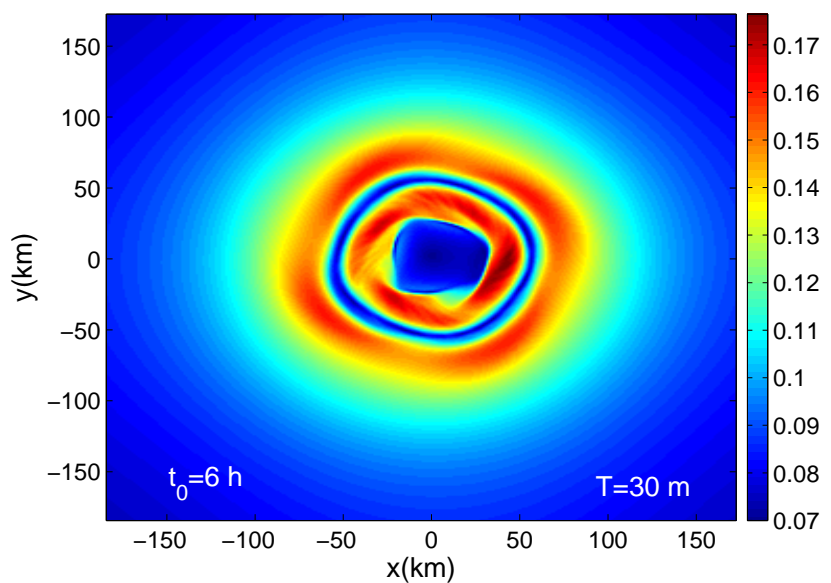

(c)

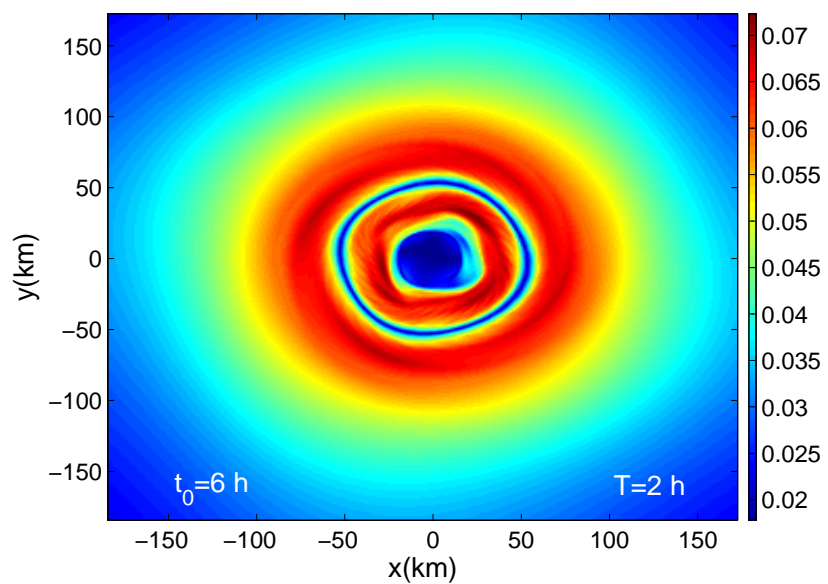

(e)

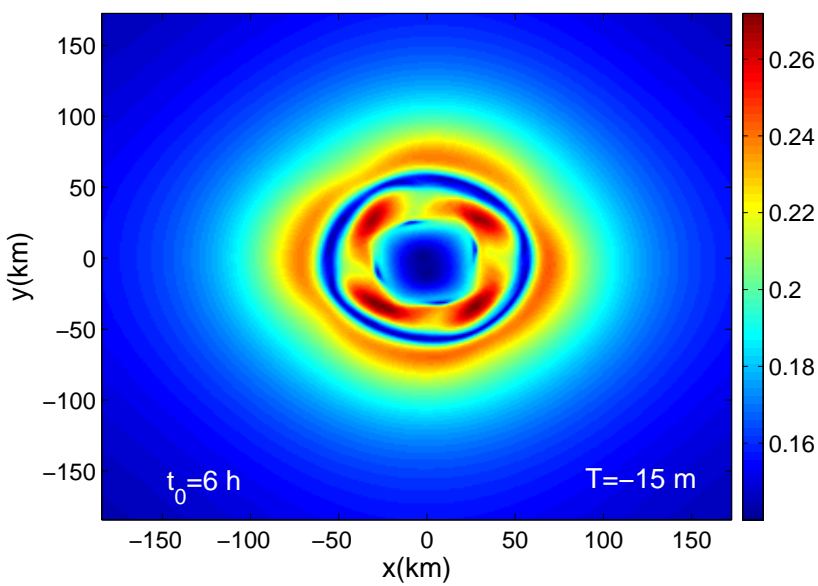

(b)

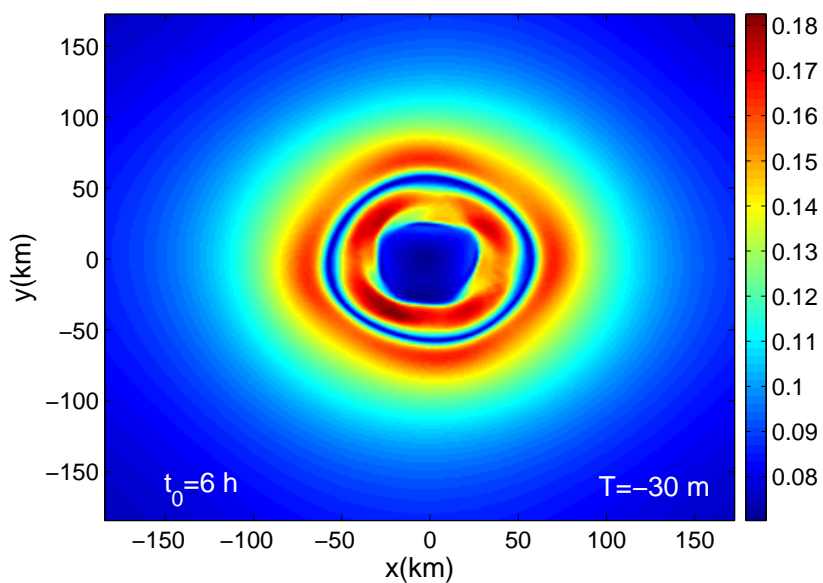

(d)

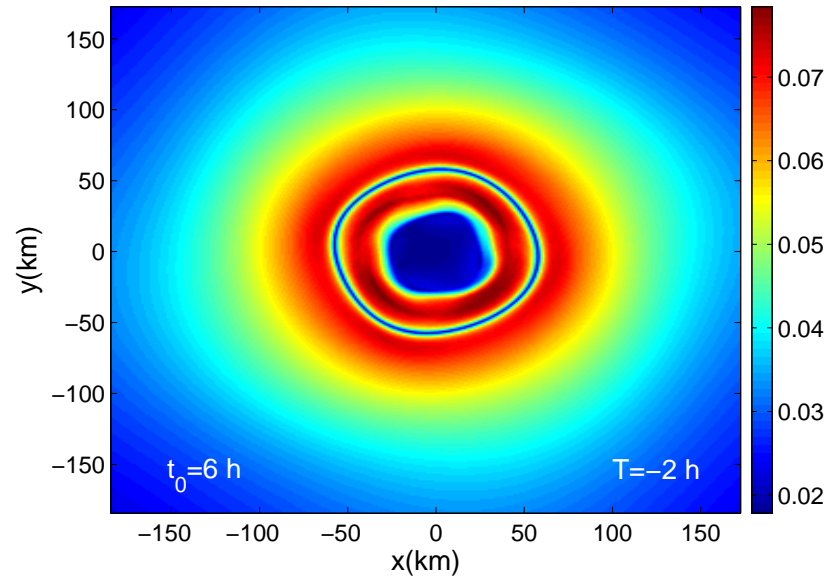

(f)

Fig. 1. Forward and backward FTLE-fields at the initial time $t_{0}=6$ hours integrated (a) $15 \mathrm{~min}$, (b) $-15 \mathrm{~min}$, (c) $30 \mathrm{~min}$, (d) $-30 \mathrm{~min}$, (e) $120 \mathrm{~min}$, and (f) $-120 \mathrm{~min}$. 


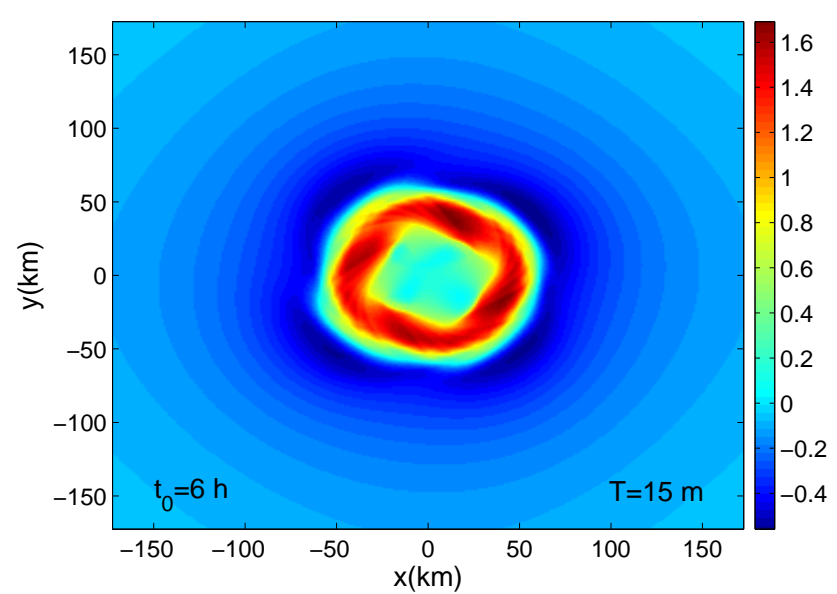

(a)

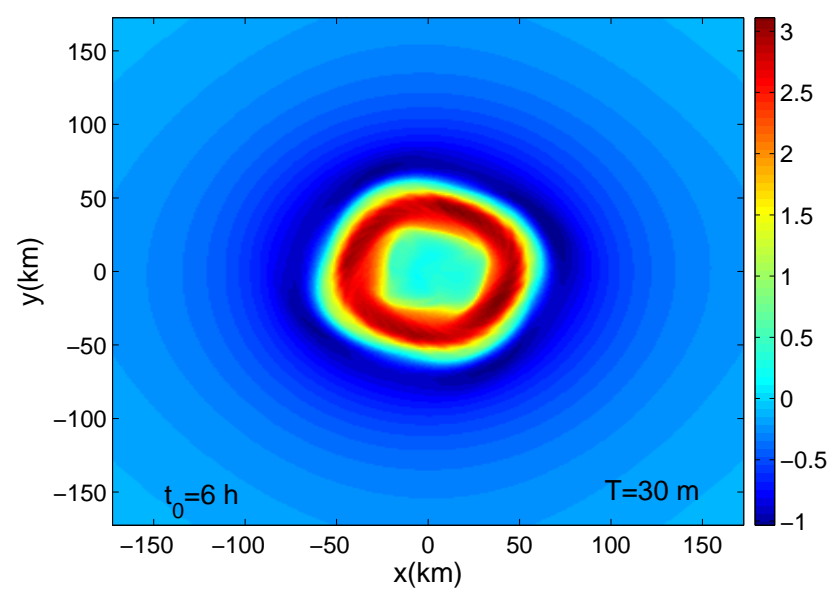

(c)

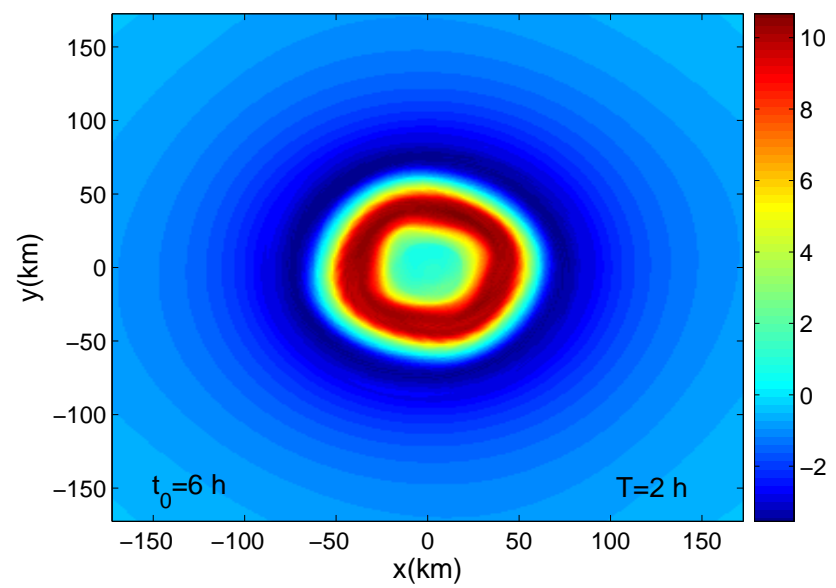

(e)

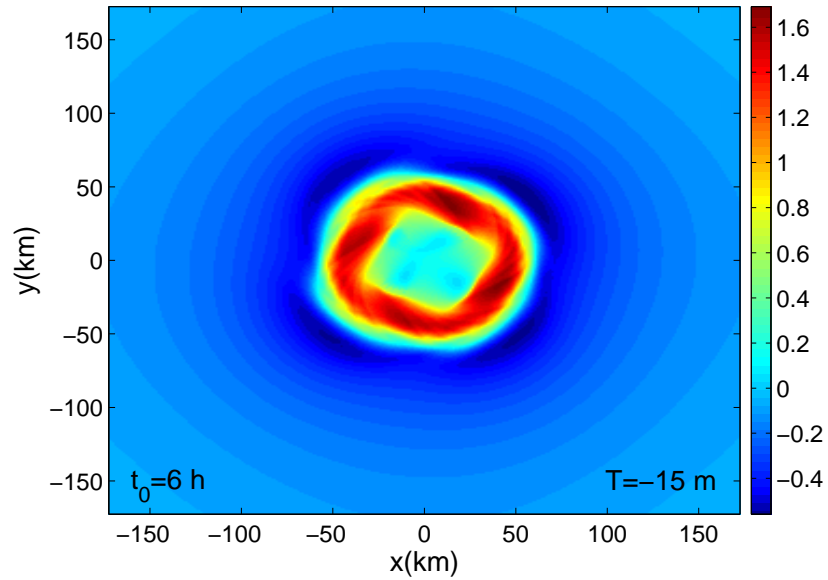

(b)

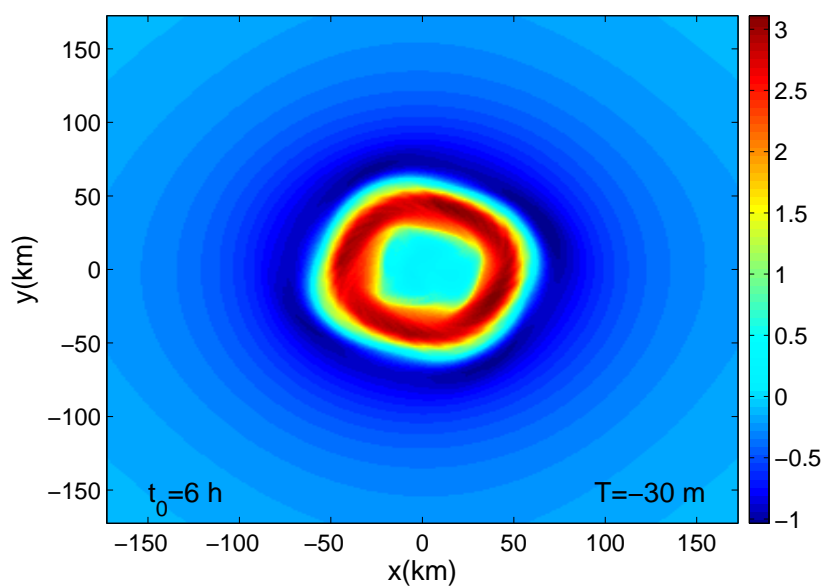

(d)

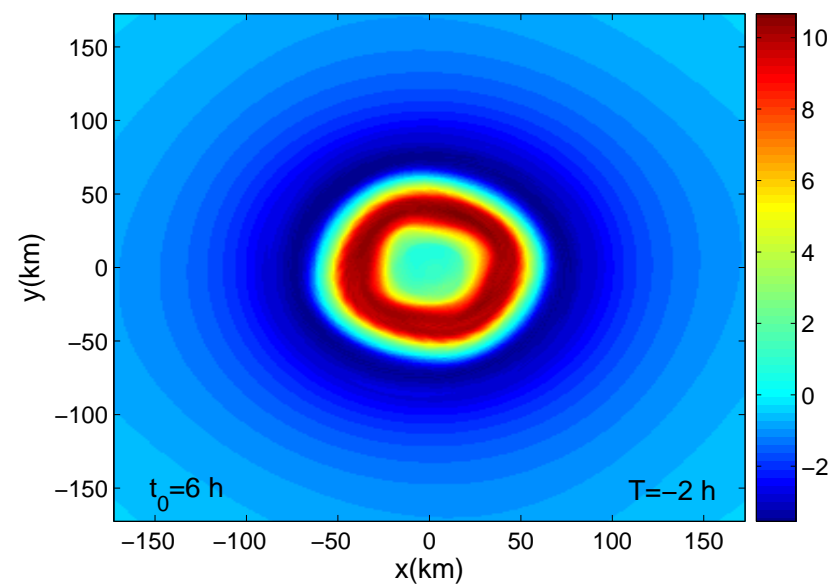

(f)

Fig. 2. Forward and backward $S$-fields at the initial time $t_{0}=6 \mathrm{~h}$ integrated (a) $15 \mathrm{~min}$, (b) $-15 \mathrm{~min}$, (c) $30 \mathrm{~min}$, (d) $-30 \mathrm{~min}$, (e) $120 \mathrm{~min}$, and (f) $-120 \mathrm{~min}$. 


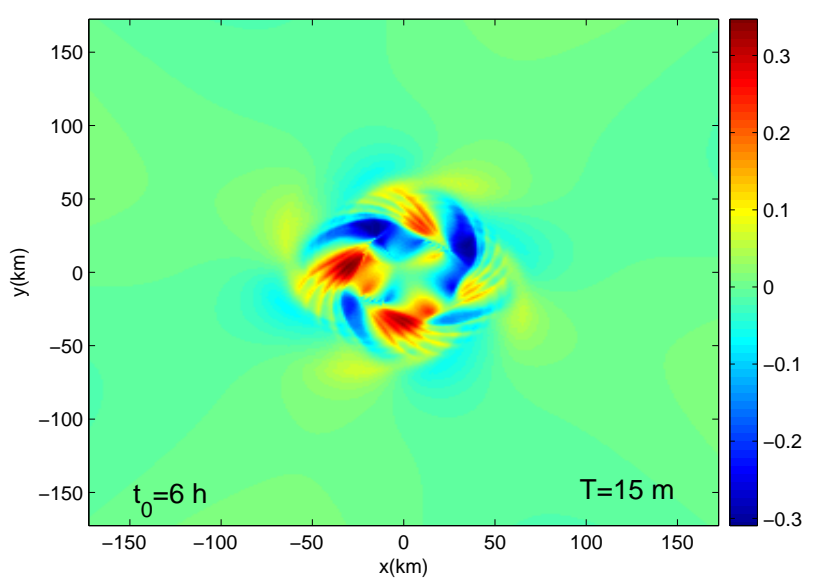

(a)

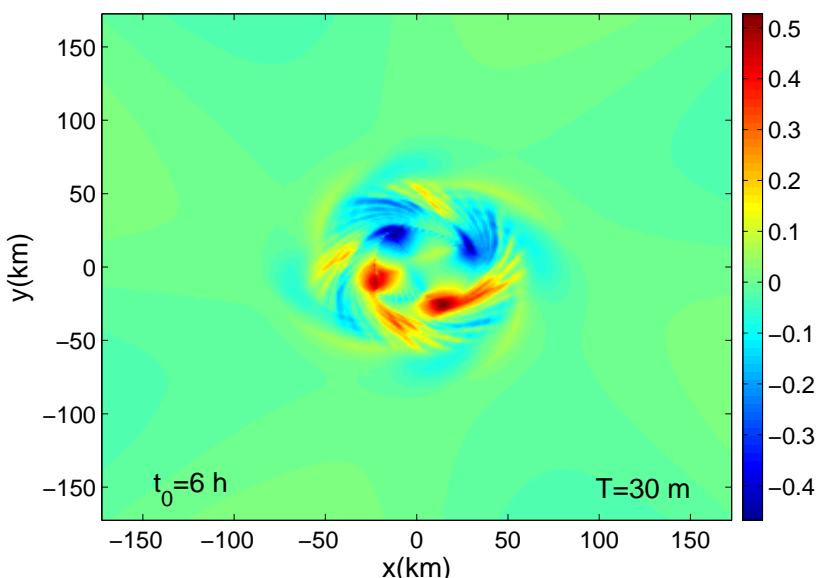

(c)

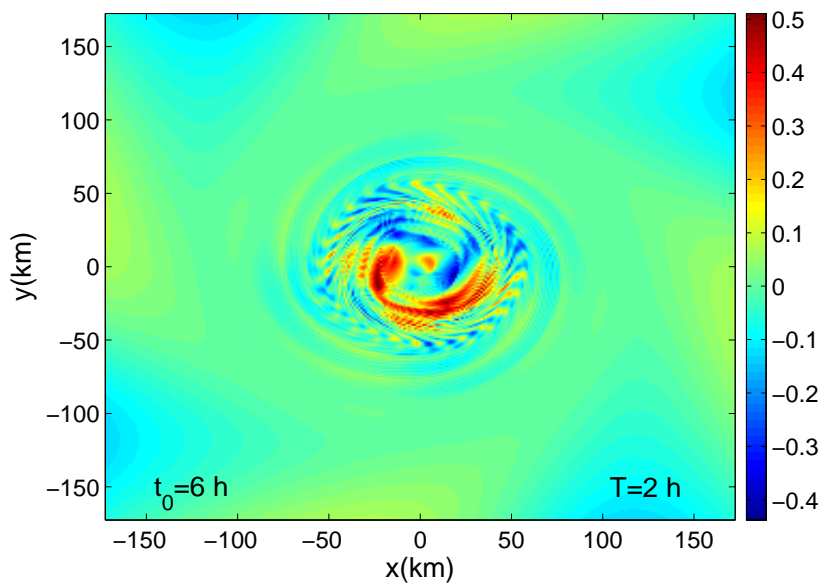

(e)

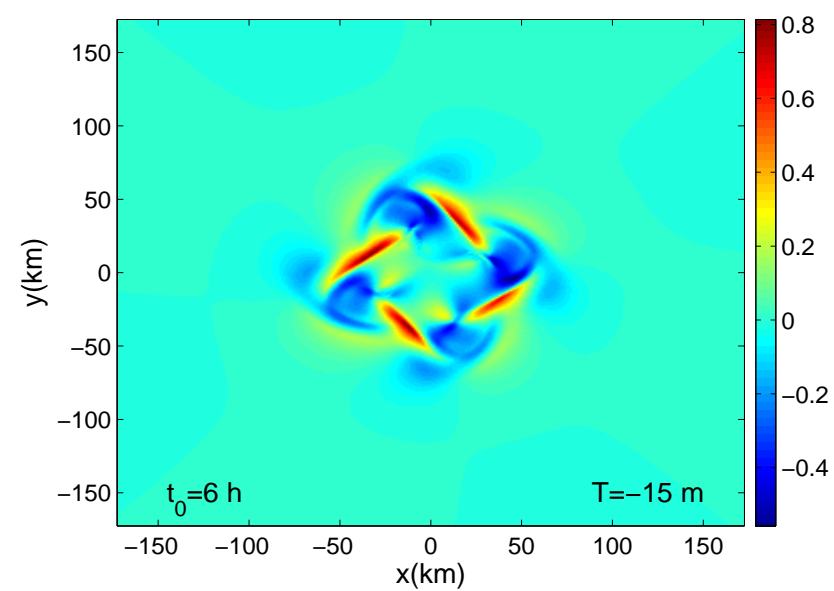

(b)

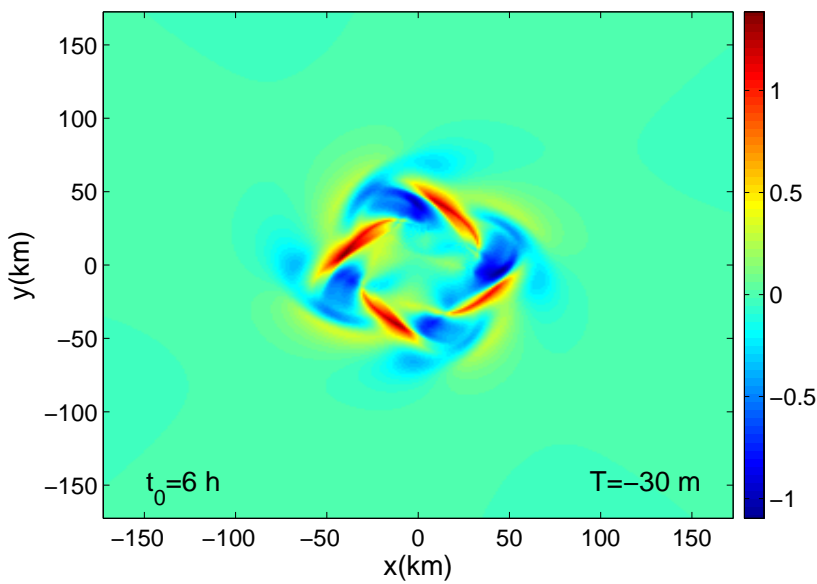

(d)

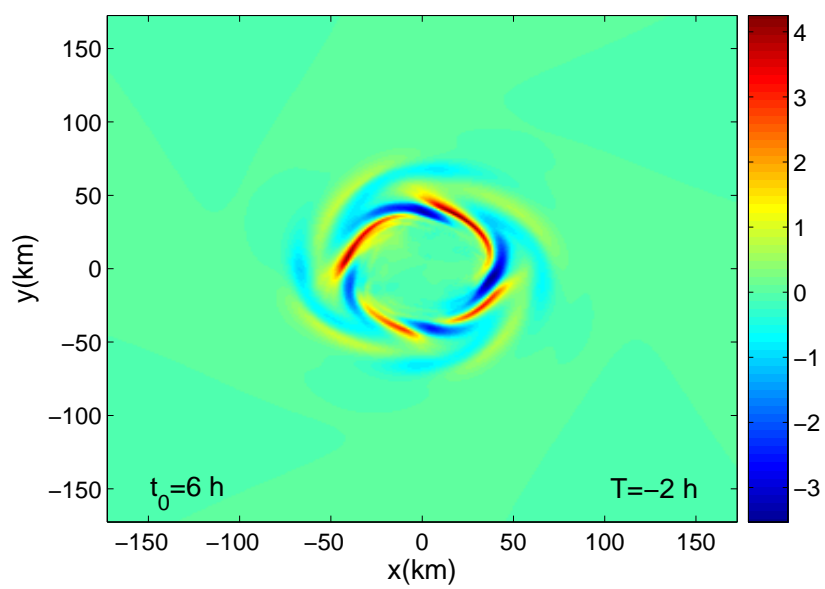

(f)

Fig. 3. Forward and backward $R$-fields at the initial time $t_{0}=6 \mathrm{~h}$ integrated (a) $15 \mathrm{~min}$, (b) $-15 \mathrm{~min}$, (c) $30 \mathrm{~min}$, (d) $-30 \mathrm{~min}$, (e) $120 \mathrm{~min}$, and (f) $-120 \mathrm{~min}$. 


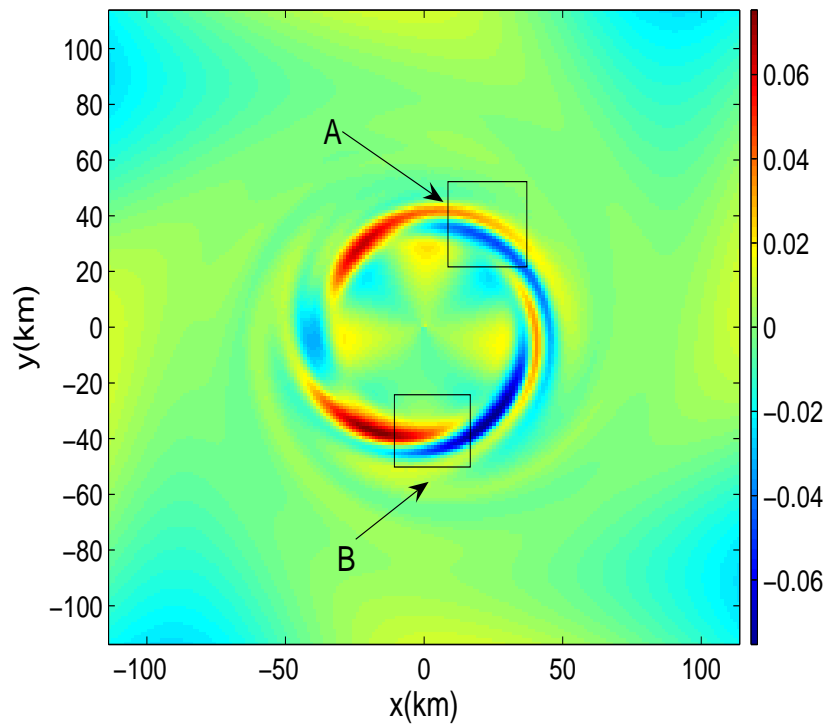

(a)

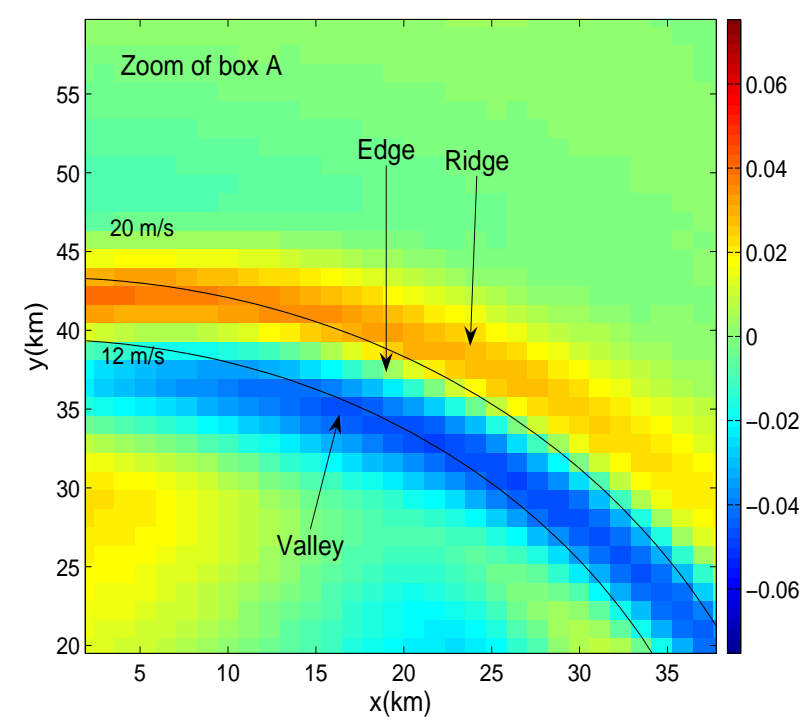

(c)

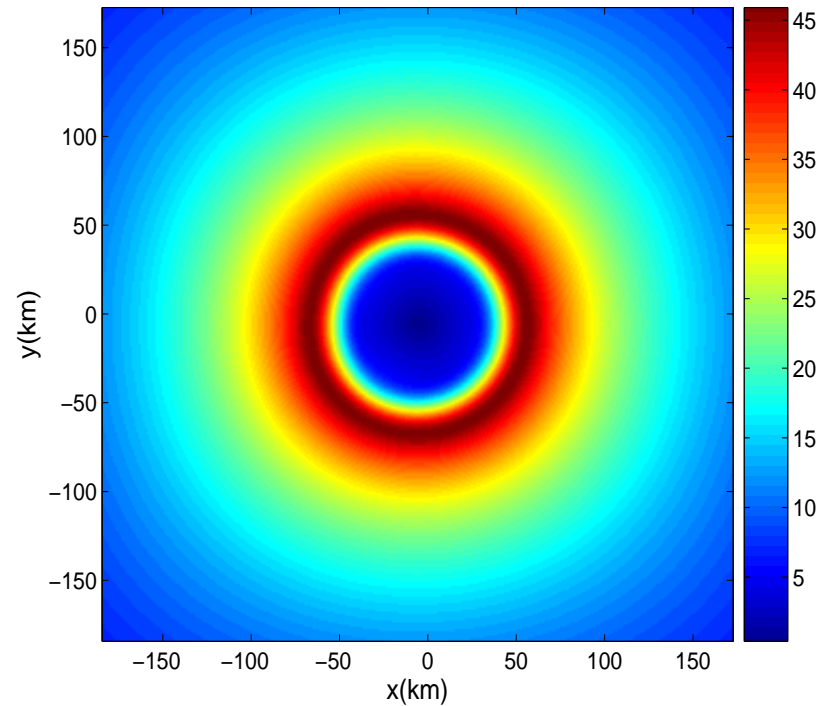

(b)

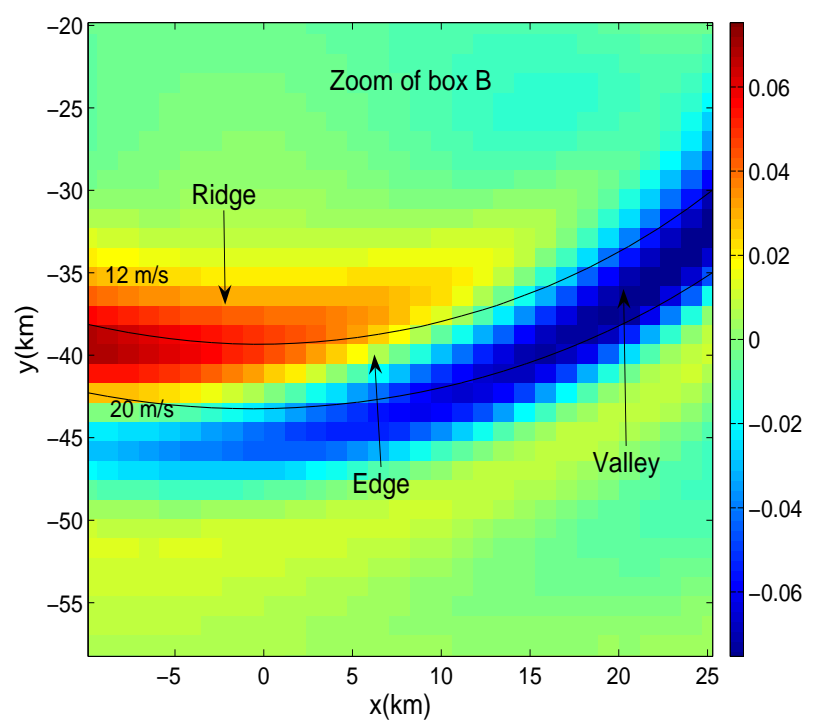

(d)

Fig. 4. (a) $R$-field with ridges and valleys and (b) Lagrangian speed at $t_{0}=2 \mathrm{~h}$. (c) Zoom of box A from $R$-field showing repelling edge, and (d) zoom of box B showing attracting edge. Integration time is $T=60 \mathrm{~min}$. Black lines show the azimuthal velocity at the initial time.

almost circular (Fig. 4b), but motion across shear-lines can be observed already.

Let $\mathcal{R}$ be a structure (ridge or valley) of $R$ at initial time $t_{0}$. The structure is coherent in the sense that it evolves continuously, for varying initial time, into a structure $\mathcal{R}^{\prime}$ seen as a ridge or valley of $R$ at initial time $t_{0}+\tau$. It is, however, not Lagrangian because it is not advected with the flow, that is, the image of $\mathcal{R}$ under the flow map, $\mathcal{R}_{\tau}=\phi_{t_{0}}^{t_{0}+\tau}(\mathcal{R})$, has advanced farther from $\mathcal{R}$ than $\mathcal{R}^{\prime}$, and is not a structure of $R$ at $t_{0}+\tau$ (Fig. 5a). Generally we observe that the coherent structures move at a slower rotational speed than that of the mean flow, which can be attributed to the effect of Rossby waves (Montgomery and Lu, 1997).

A coherent structure $\mathcal{R}$ has a leading and a lagging end relative to counterclockwise rotation. Concerning the evolution of $\mathcal{R}$ under the flow map, two cases can occur for structures computed in a forward time integration:

(a) If the leading end is at higher angular velocity than the lagging end, then the image $\mathcal{R}_{T}$ of $\mathcal{R}$ under $\phi_{t_{0}}^{t_{0}+T}$ is lengthened over the integration and tends to align with a contour of the $S$-field (Fig. 5b). 
(b) If the leading end is at lower angular velocity than the lagging end, then the flow map rotates $\mathcal{R}$, and for sufficiently large $T$ the image $\mathcal{R}_{T}$ tends to align with a contour of the $S$-field in the opposite direction (Fig. 5c).

For a nearby pair of a ridge and a valley, the relative position of their flow map images is preserved in case a, whereas in case $\mathrm{b}$ they switch position. This rotation and position switching are a mechanism for the advective mixing during the polygonal eyewall stage. The square eyewall gives four valleys and ridges aligned in a way that four sets of trajectories pass from outside to inside and four from inside to outside of an $S$-contour. The combination of ridge-edgevalley sets of $R$, aligned with the leading ends at lower angular velocity, can be seen as an indicator of fluid regions that will roll into mesovortices over the forward integration time. Thus the strength and size of the surrounding ridges and valleys are an indicator of the potential flux in and out of the mesovortex.

\section{Field diagnostics for varying initial time}

In Figs. 6 to 11 we show in panel a the relative vorticity, in panel b the $Q$-field, and in panel c the $R$-field with overlayed vorticity contours chosen to illustrate the relation between $R$-field structures, and vorticity structures. We show the $S$-field, integrated $Q$-field, and FTLE field in panel d, $\mathrm{e}$, and $\mathrm{f}$ respectively, together with contours (from inside to outside) of the maximum normal propogating shear, the maximum tangential velocity, and the maximum counterpropogating shear. The initial times in these figures are $t_{0}=2 \mathrm{~h}$, $4 \mathrm{~h}, 6 \mathrm{~h}, 8 \mathrm{~h}, 10 \mathrm{~h}$, and $12 \mathrm{~h}$, and the integration time is $T=1 \mathrm{~h}$.

Since the shear-lines are distorted circles, we can interpret the average $\bar{\sigma}\left(r_{0}, t_{0}\right)\left(r_{0}=\left|\mathbf{x}_{0}\right|\right)$ as radial mixing rate. In all Figs. 6 to $11 \mathrm{~d}-\mathrm{f}$ we observe that extreme $\widehat{Q}, S$, and FTLEvalues occur at extrema of $\overline{\partial \omega / \partial r}$, demonstrating that the $\widehat{Q}$, $S$, and FTLE-fields are dominated by the shear. A similar interpretation as radial mixing rates can be attributed to the averages $\bar{S}\left(r_{0}, t_{0}\right)$ and $\bar{R}\left(r_{0}, t_{0}\right)$. Plots of $\bar{S}$ and $\bar{\sigma}$ reveal these two averages are very similar in structure, as both measure shear. The quantity $\bar{R}$ can be interpreted as a measure of hyperbolic mixing, which is important for transport through the eyewall.

\section{$5.12-4$ h: initial state}

At the initial time of $2 \mathrm{~h}$ (Fig. 6), the model is still close to the initial state and shows a broad ring of high vorticity fluid. While the vorticity, $Q, S$, and FTLE-fields are almost circular-symmetric, the $R$-field shows distinct lines of high radial mixing, demostrating that coherent structures can persist through dominant shear. The wavenumber four asymmetry begins to show in the $R$-field, particularly in the forward time integration, atthough the initial vorticity profile is nearly preserved, with any asymmetries barely noticible. The

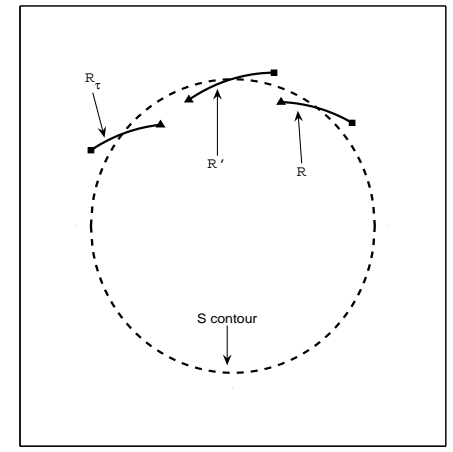

(a)

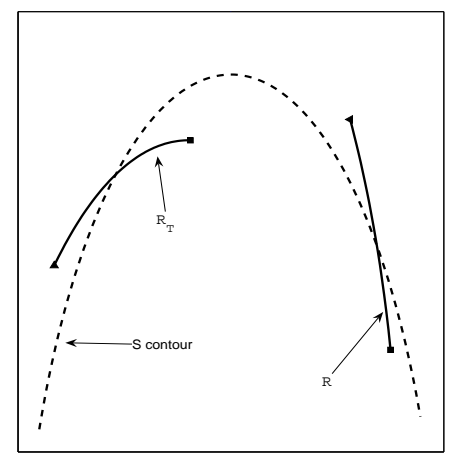

(b)

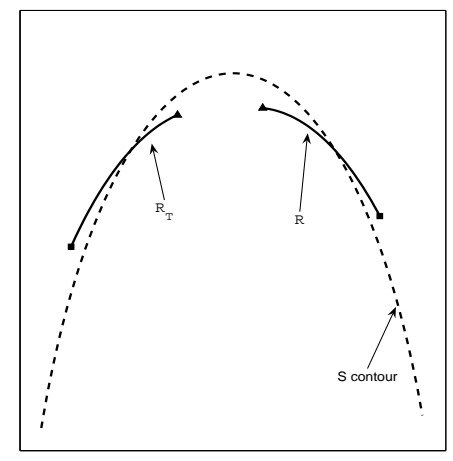

(c)

Fig. 5. (a) Sketch of a structure $\mathcal{R}$ of $R$ at initial time $t_{0}$, the associated structure $\mathcal{R}^{\prime}$ of $R$ at initial time $t_{0}+\tau$, and the flow map image $\mathcal{R}_{\tau}=\phi_{t_{0}}^{t_{0}+\tau}(\mathcal{R})$, illustrating the non-Lagrangian nature of the coherent structures. (b) Structure $\mathcal{R}$ crossing an $S$ contour with speed above the contour higher than the speed below, and flow map image $\mathcal{R}_{T}$ after an integration time. Leading and lagging ends of $\mathcal{R}$ and their images on $\mathcal{R}_{T}$ are marked by a triangle and a square, respectively. (c) Same as (b) with opposite orientation of $\mathcal{R}$ relative to the contour, leading to a rotation of $\mathcal{R}_{T}$. 


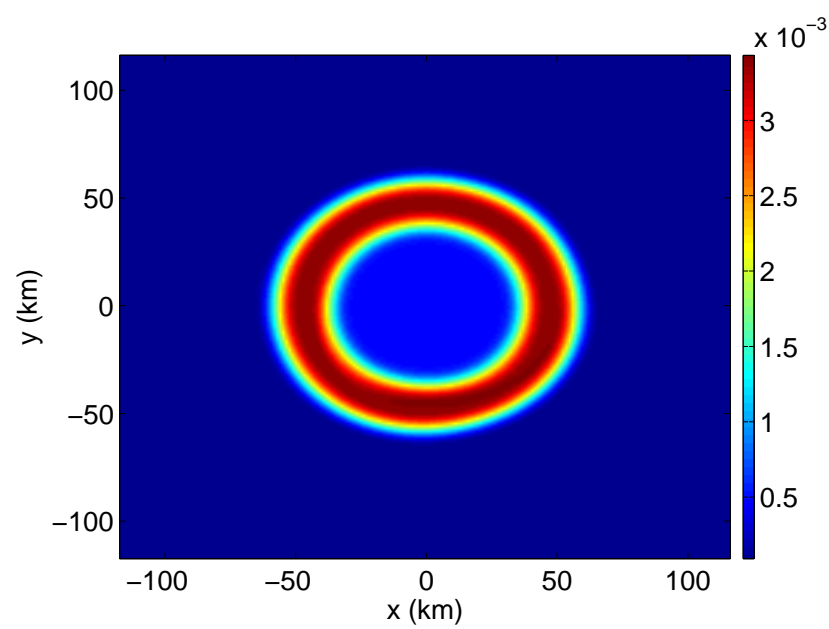

(a)

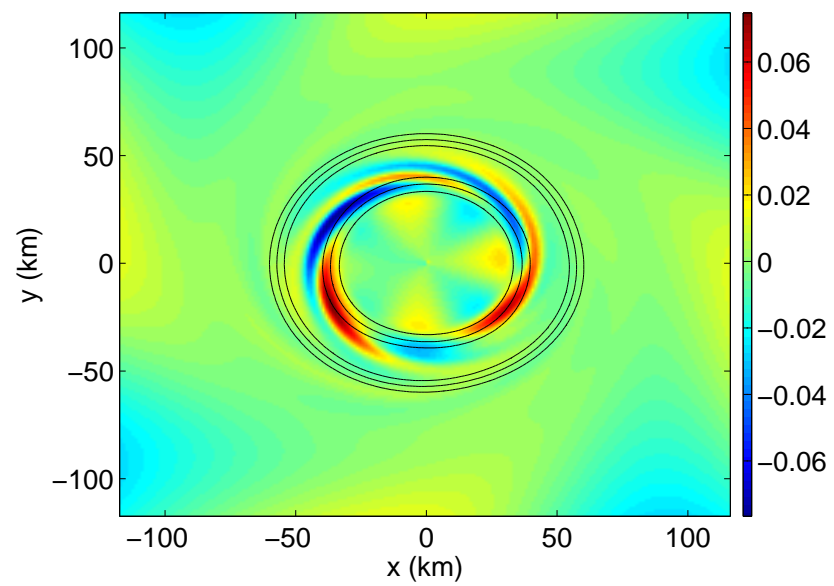

(c)

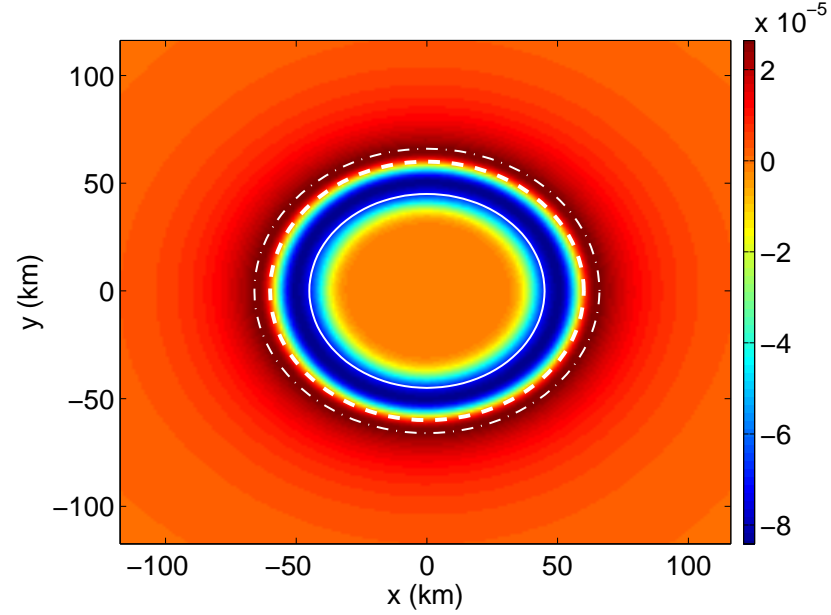

(e)

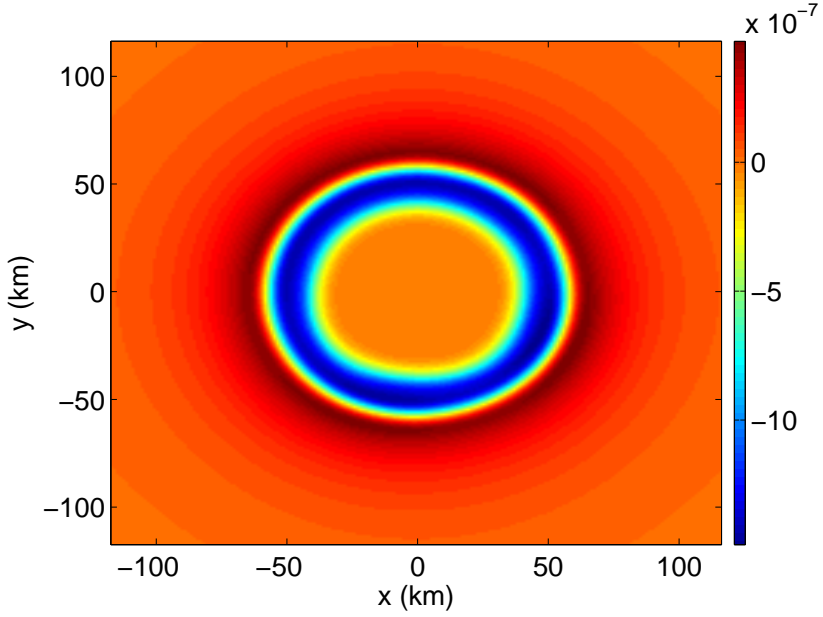

(b)

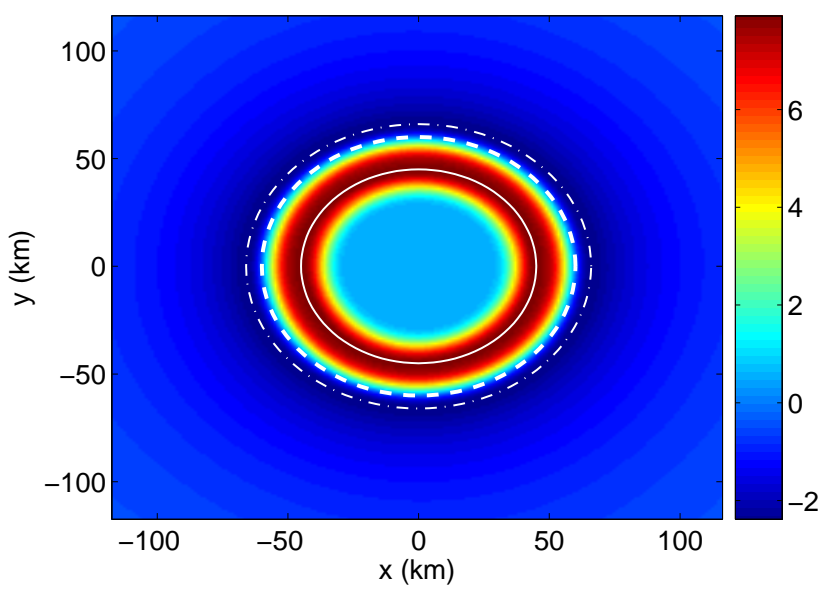

(d)

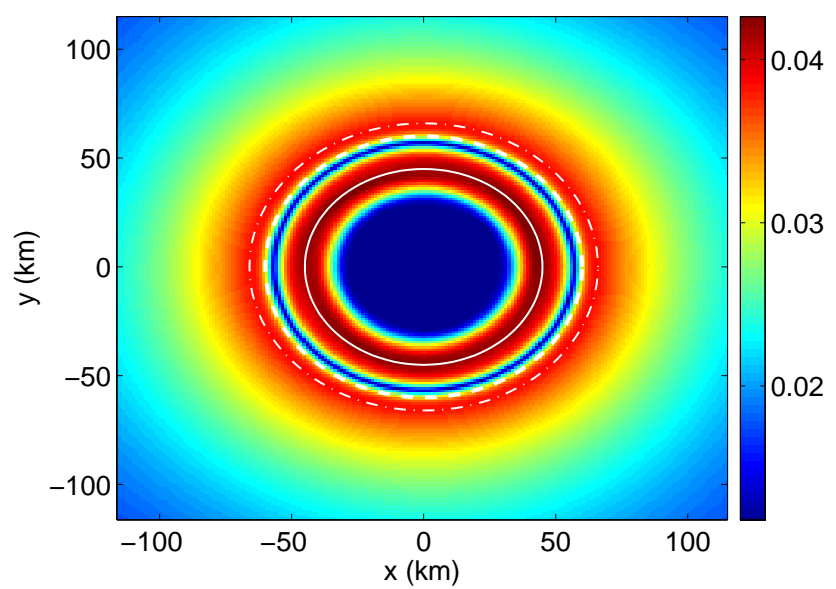

(f)

Fig. 6. (a) Relative vorticity field, (b) $Q$-field, (c) $R$-field with vorticity contours overlayed to relate structures, (d) $S$-field, (e) integrated $Q$-field $\widehat{Q}$, and (f) $F T L E$-field for initial time of $t_{0}=2 \mathrm{~h}$ with integration time $T=1 \mathrm{~h}$. The white contours in (d)-(f) mark the radius of maximum $\frac{\partial \omega}{\partial r}$ (inner, solid), the radius of maximum tangential wind (middle, dashed), and the radius of maximum counterpropogating $\frac{\partial \omega}{\partial r}$ (outer, dash-dot) 
rotation of the $R$-ridges and valleys in this stage allows the crystallization that is neccessary for mesovortex formation.

At $4 \mathrm{~h}$ (Fig. 7), much of the symmetry of the initial state still remains. A forward time integration begins to display asymmetries in the $Q$, FTLE- and $S$-fields, whereas the $R$ field retains the structures that were present at $2 \mathrm{~h}$. At $4 \mathrm{~h}$ the backward time integration of $R$ (not shown) also shows regions of high orthogonal separation.

\section{$5.26-8 \mathrm{~h}$ : polygonal eyewall}

At $6 \mathrm{~h}$ (Fig. 8), the vorticity field shows a polygonal eyewall structure, where the flow resembles a nonlinear critical layer for dry barotropic instability. A square inner eyewall structure forms, with pools of low vorticity fluid organized into the corners of the eye. The fluid is largely unmixed, with low vorticity fluid organized in the mesovortices, where it is largely protected from the outer flow. Since vorticity is materially conserved, low vorticity fluid from the eye and environment must replace the fluid that left the eyewall. The fluid that is mixed across the boundaries is consistent with the ridges and valleys of the $R$-field. The filamentation that develops from the stretching of high vorticity fluid that exits from the vorticity ring to the environment, can be seen in the form of spiral bands in the vorticity field.

The $Q$-field shows that, instantaneously, high trajectory separation occurs along the square boundary of the inner eyewall. The pools of low vorticity show instantaneous contraction. The outer vorticity ring shows high trajectory separation. Even when the FTLE-fields are calculated for the small integration time of $T=3 \mathrm{~min}$, there is a noticible difference between the separation points of the $Q$-field and the $F T L E$-field. The square eyewall formation corresponds to four structures of high FTLE-values in both the forward and backward time fields for short integration times. As integration time is increased, the structures lengthen and are no longer distinguishable.

The $R$-field shows a series of ridges and valleys that originated as coherent structures from the earlier times, but are not as refined as previous structures. There are also structures emanating outward from the ring of high vorticity that may play the role of protecting the ring from interaction with the outer flow (Dunkerton et al., 2009). If our model was a true representation of a wave critical layer, the structures would correspond to dividing streamlines, but the high shear prohibits this. The maximal $R$-regions are located at the same places that show high FTLE-values, but with much greater resolution than the FTLE-fields, which are blurred by the shear.

At $8 \mathrm{~h}$ (Fig. 9), the polygonal eyewall structure that is present at $6 \mathrm{~h}$ is still clearly visible. The $R$-field has similar properties as the $R$-field at $6 \mathrm{~h}$ (Fig. 8), with the coherent structures begining to merge, showing intense mixing.

\subsection{Later state: mixing into a monopole}

The period after $t_{0}=8 \mathrm{~h}$ until $t_{0}=12 \mathrm{~h}$ exhibits intense mixing that leads to a collapse into a monopole end state.

For $t_{0}=10 \mathrm{~h}$ (Fig. 10), the $Q$-field is less square, and the $R$-field shows high mixing in two distinct regions, one with expansion and one with contraction. The region of expansion is inside the ring of high vorticity, while the region of contraction has become organized in the dominant mesovortex, which is the "winner" and survives to become the primary vortex during the collapse into the monopole end state. Note that the merging of LCS's into a single LCS is a bifurcation, and cannot happen if they represent true stable and unstable manifolds, however the coherent structures here are not entirely Lagrangian, yet their interactions and bifurcations play an important role in the systematic mixing during mesovortex interaction. As more advection (stirring) in and out of the eyewall occurs, there is filamentation of the initial vorticity-contours with diffusive mixing occurring along the lengthening contour boundaries, leading to an "averaging" of vorticity values through diffusion.

For $t_{0}=12 \mathrm{~h}$ (Fig. 11), the inner ring of vorticity has broken down. The FTLE- and $S$-fields show the outer rings converged as a thick ring, and the model is entering the monopole state. The $R$-field coherent structures now show the dominant mesovortex migrating to the center and the other mesovortices are disappearing due to their annihilation by the dominant mesovortex. Regions of high $R$-values are pushed outward, indicationg mixing with the outer flow. At this stage, there is a single remaining protecting $R$-ridge, on the outside of the remaining high vorticity ring, which has served the role of protecting the mesovortex that eventually becomes the "winner".

Beyond $12 \mathrm{~h}$, the initial regions of vorticity are not recognizable, and high (although not as high as the initial state) vorticity fluid begins to organize into the eye. The low vorticity fluid from the eye becomes well mixed, and the eyewall and environment become filled with relatively low vorticity fluid. The angular velocity gradient decreases, and the $S$ field shows no eyewall.

Although the fluid that is mixed beyond $12 \mathrm{~h}$ is not distinguishable based on its initial vorticity, the $R$-field still gives regions of advective mixing, showing that the moving frame of initial conditions still shows regions of fluid that are transported.

\section{Mixing rates}

The radial mixing rates $\bar{\sigma}\left(r_{0}, t_{0}\right)$ and $\bar{S}\left(r_{0}, t_{0}\right)$ quantify mixing due to shear, whereas $\bar{R}\left(r_{0}, t_{0}\right)$ quantifies hyperbolic mixing. In these mixing rates, the lines along and across which mixing is quantified are circles. Hyperbolic mixing rates that are more closely related to the shearing structures are mixing 


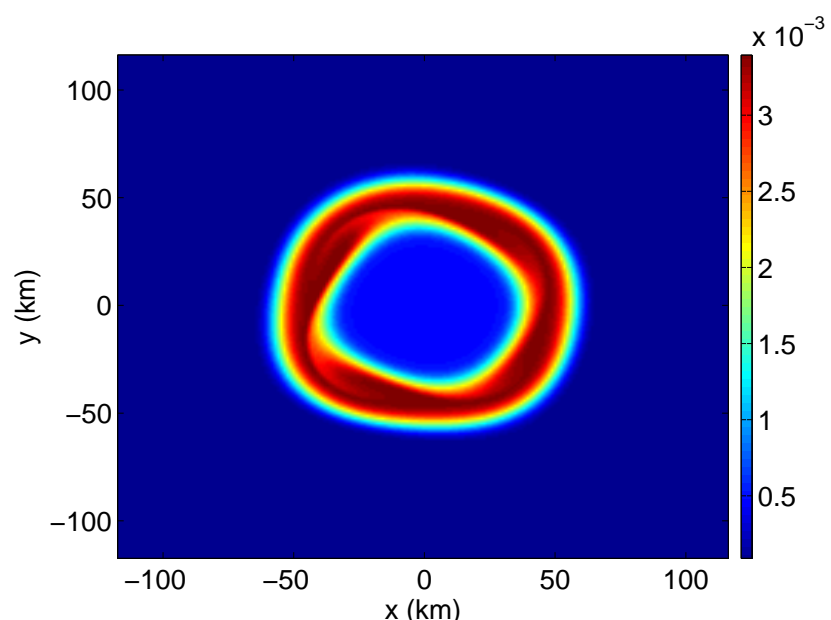

(a)

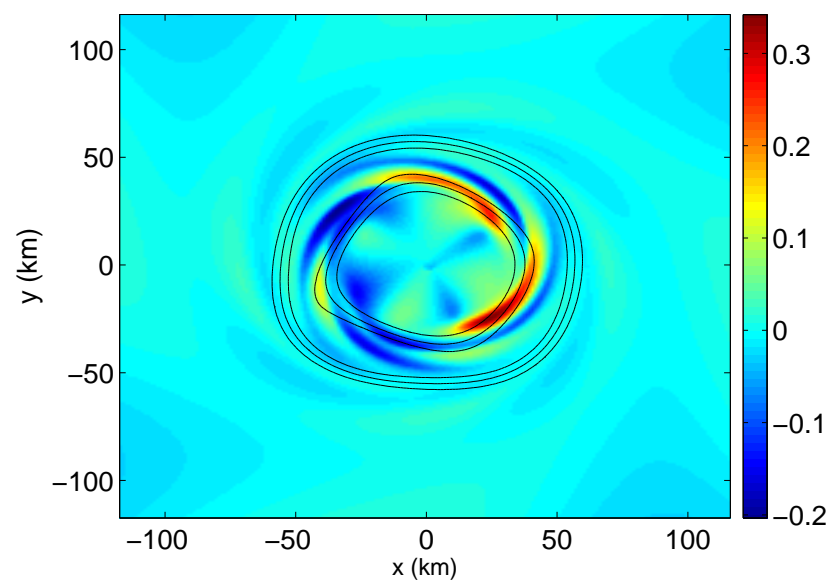

(c)

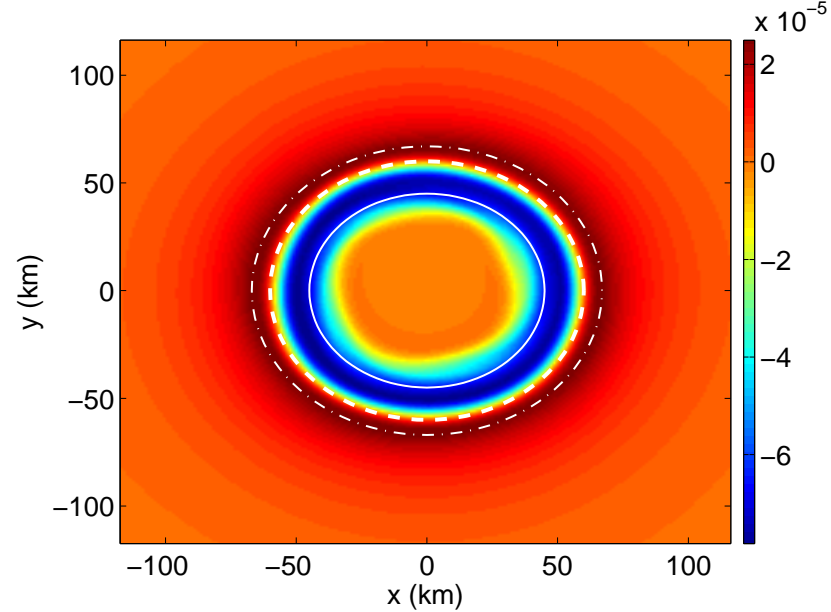

(e)

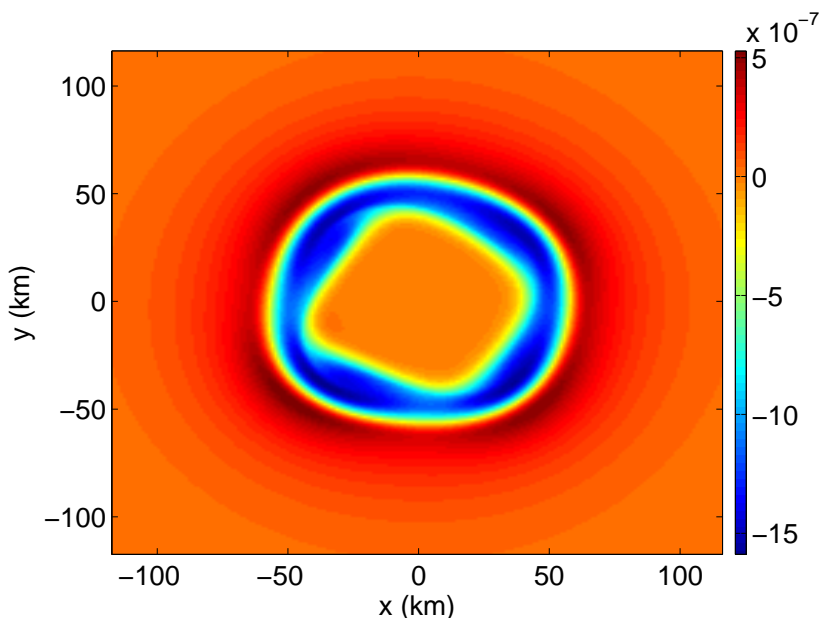

(b)

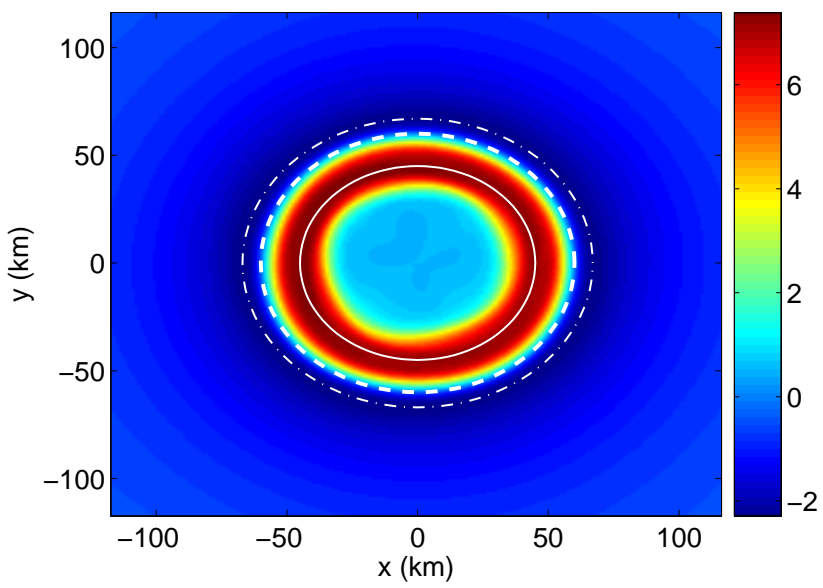

(d)

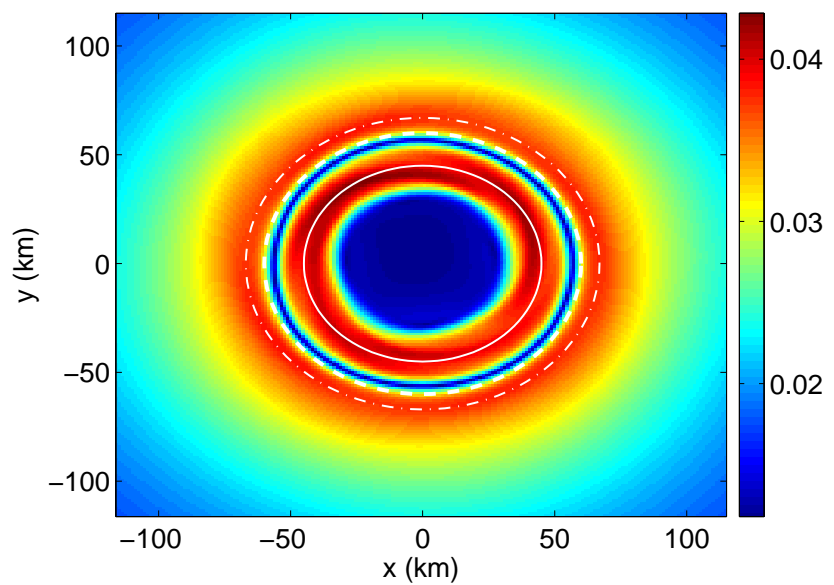

(f)

Fig. 7. (a) Relative vorticity field, (b) $Q$-field, (c) $R$-field with vorticity contours overlayed to relate structures, (d) $S$-field, (e) integrated $Q$-field $\widehat{Q}$, and (f) $F T L E$-field for initial time of $t_{0}=4 \mathrm{~h}$ with integration time $T=1 \mathrm{~h}$. The white contours in (d)-(f) mark the radius of maximum $\frac{\partial \omega}{\partial r}$ (inner, solid), the radius of maximum tangential wind (middle, dashed), and the radius of maximum counterpropogating $\frac{\partial \omega}{\partial r}$ (outer, dash-dot). 


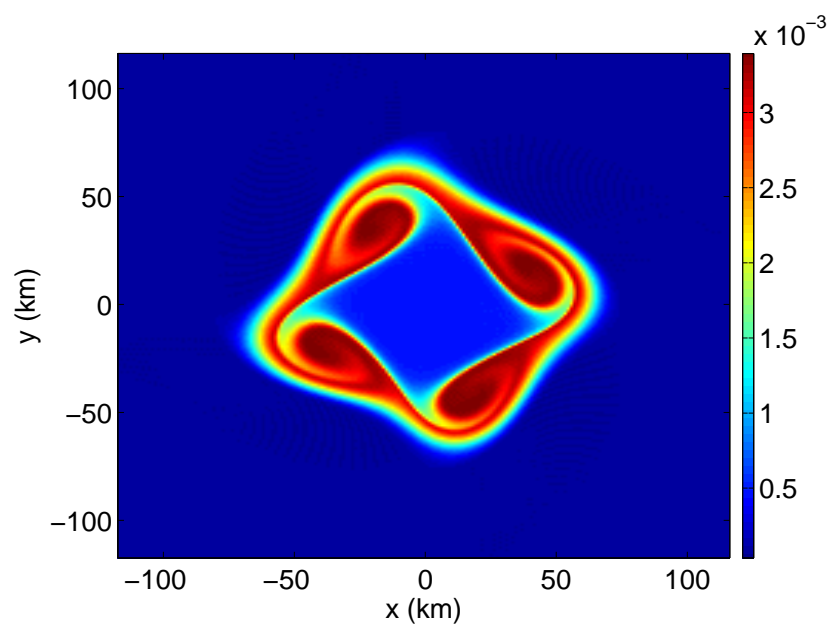

(a)

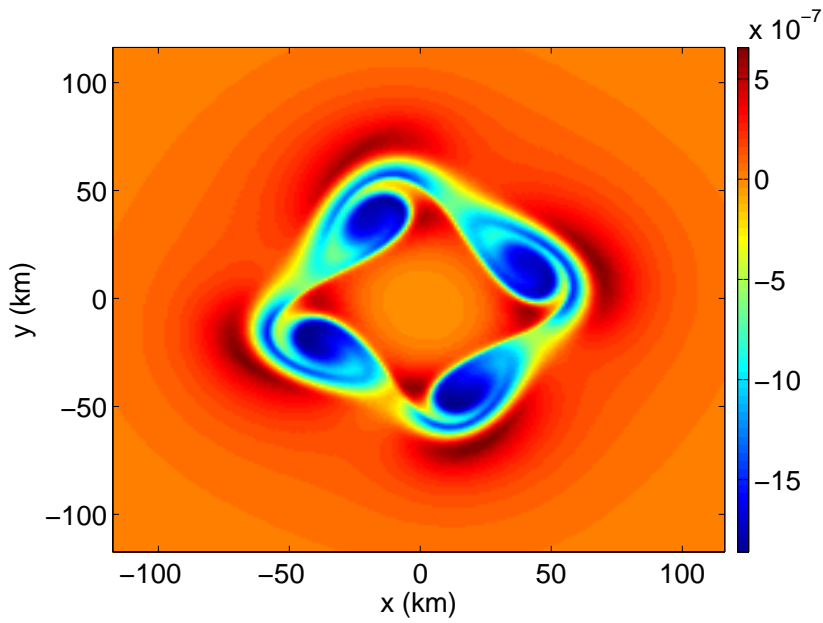

(b)

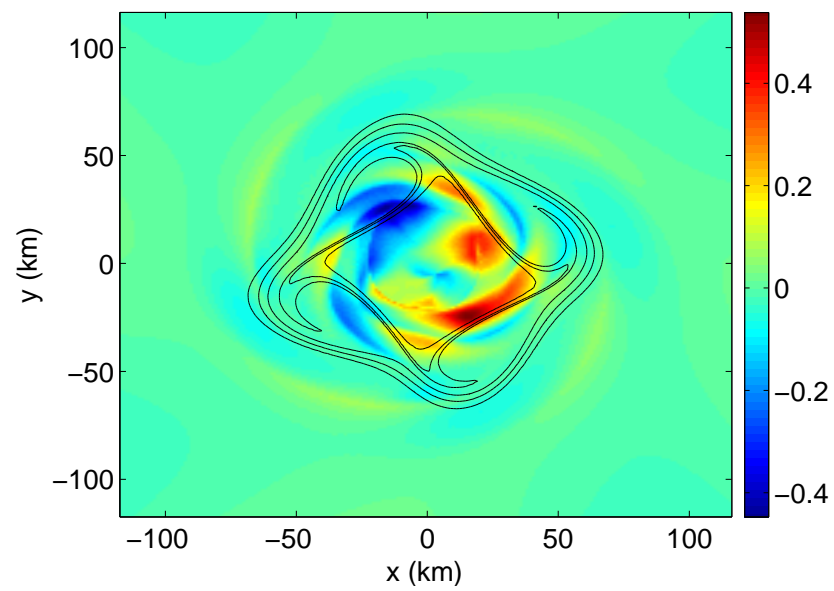

(c)

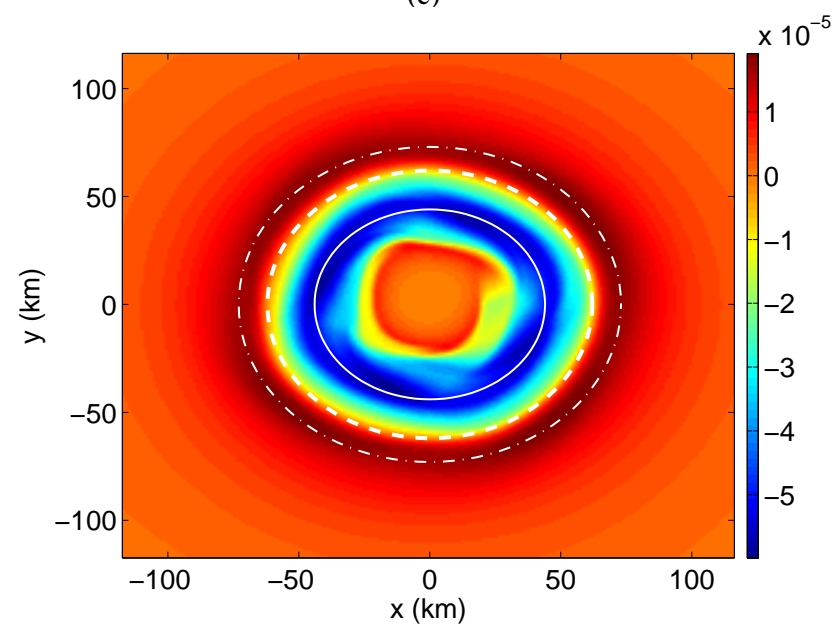

(e)

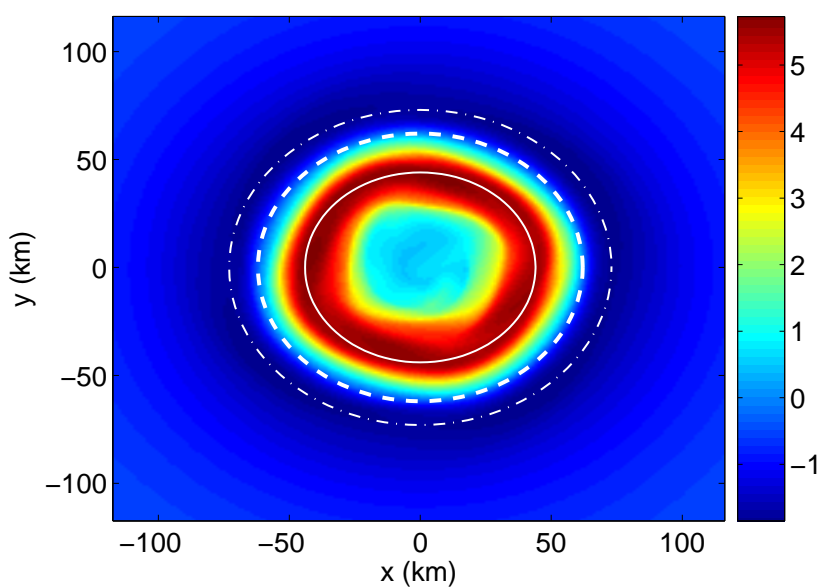

(d)

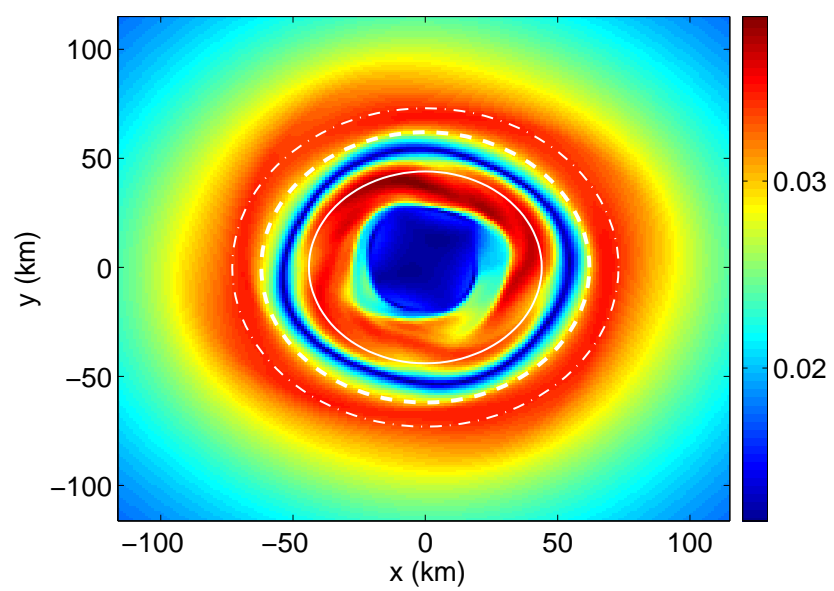

(f)

Fig. 8. (a) Relative vorticity field, (b) $Q$-field, (c) $R$-field with vorticity contours overlayed to relate structures, (d) $S$-field, (e) integrated $Q$-field $\widehat{Q}$, and (f) $F T L E$-field for initial time of $t_{0}=6 \mathrm{~h}$ with integration time $T=1 \mathrm{~h}$. The white contours in (d)-(f) mark the radius of maximum $\frac{\partial \omega}{\partial r}$ (inner, solid), the radius of maximum tangential wind (middle, dashed), and the radius of maximum counterpropogating $\frac{\partial \omega}{\partial r}$ (outer, dash-dot). 


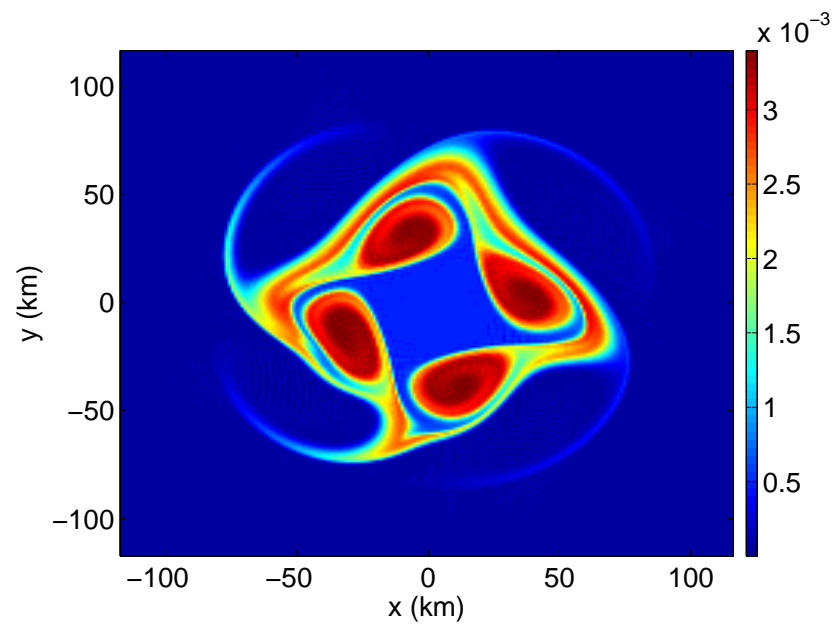

(a)

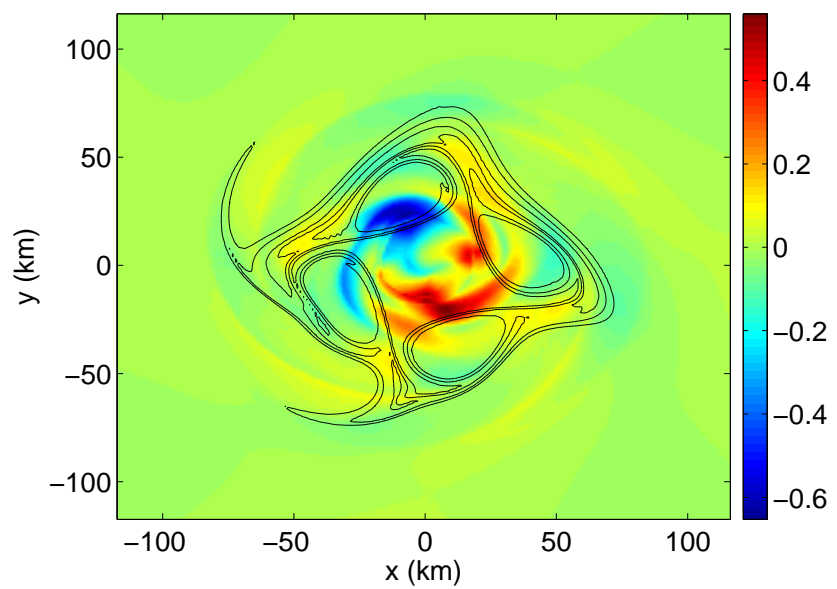

(c)

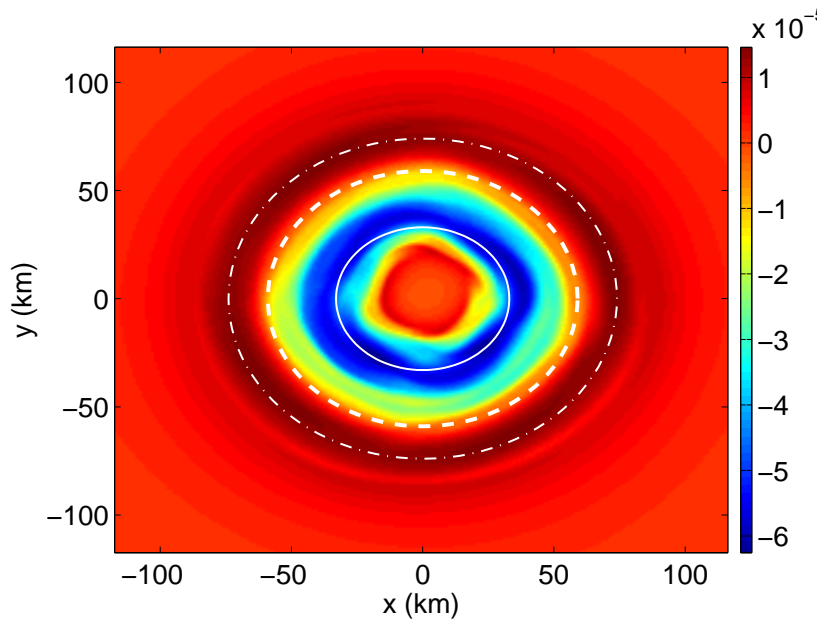

(e)

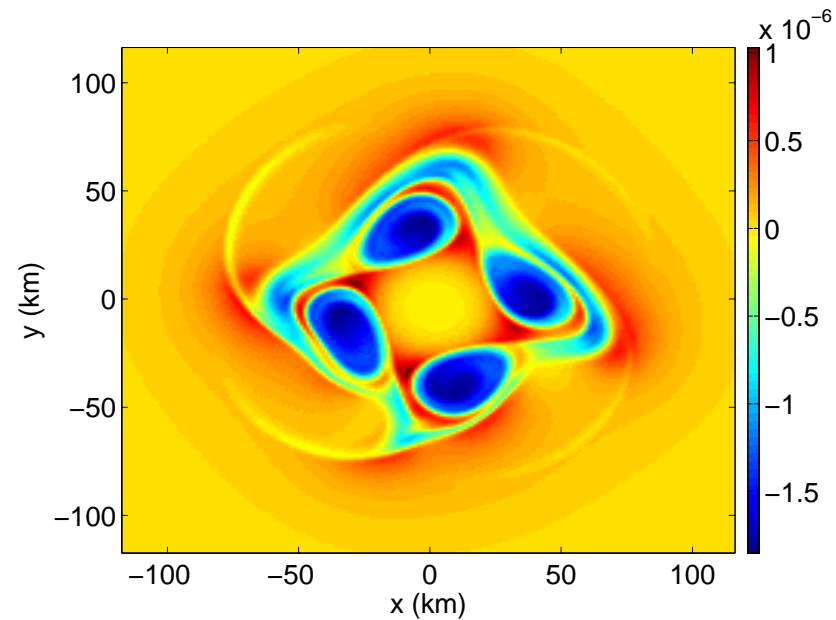

(b)

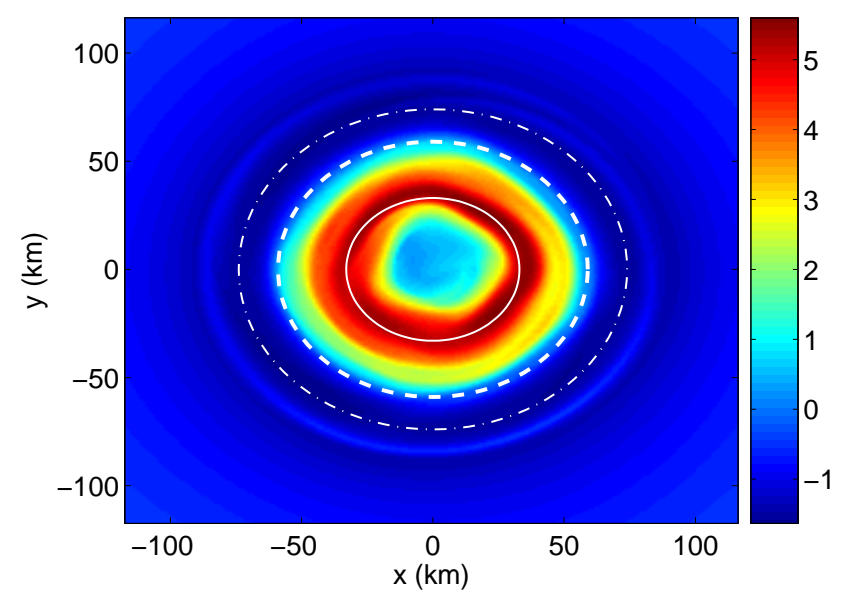

(d)

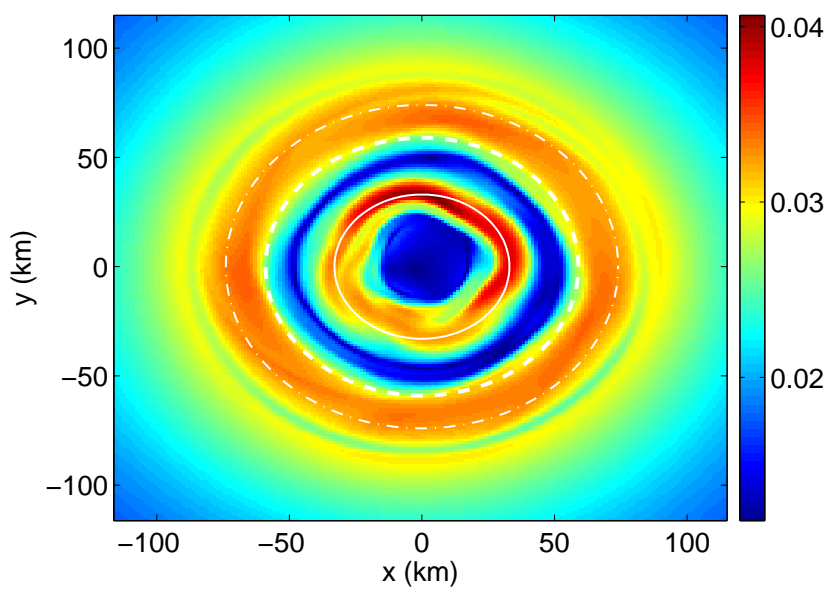

(f)

Fig. 9. (a) Relative vorticity field, (b) $Q$-field, (c) $R$-field with vorticity contours overlayed to relate structures, (d) $S$-field, (e) integrated $Q$-field $\widehat{Q}$, and (f) $F T L E$-field for initial time of $t_{0}=8 \mathrm{~h}$ with integration time $T=1 \mathrm{~h}$. The white contours in (d)-(f) mark the radius of maximum $\frac{\partial \omega}{\partial r}$ (inner, solid), the radius of maximum tangential wind (middle, dashed), and the radius of maximum counterpropogating $\frac{\partial \omega}{\partial r}$ (outer, dash-dot). 


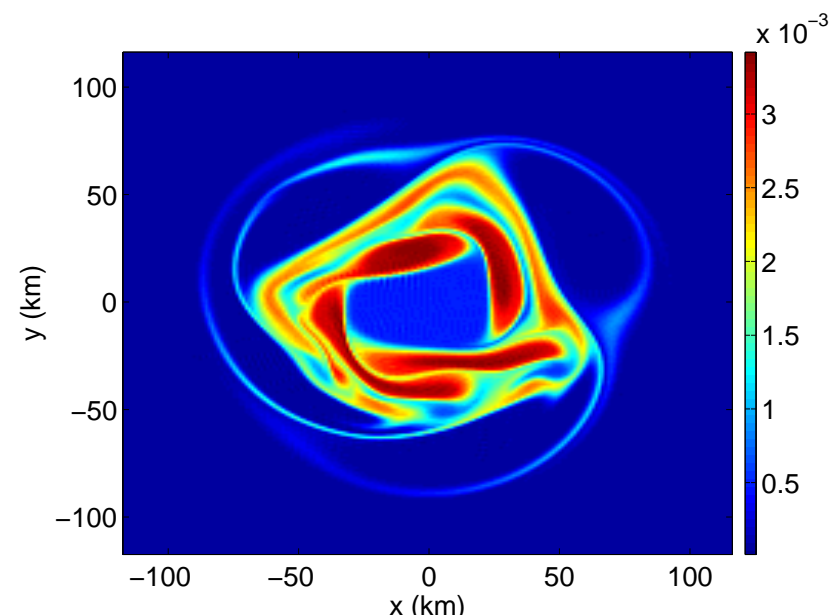

(a)

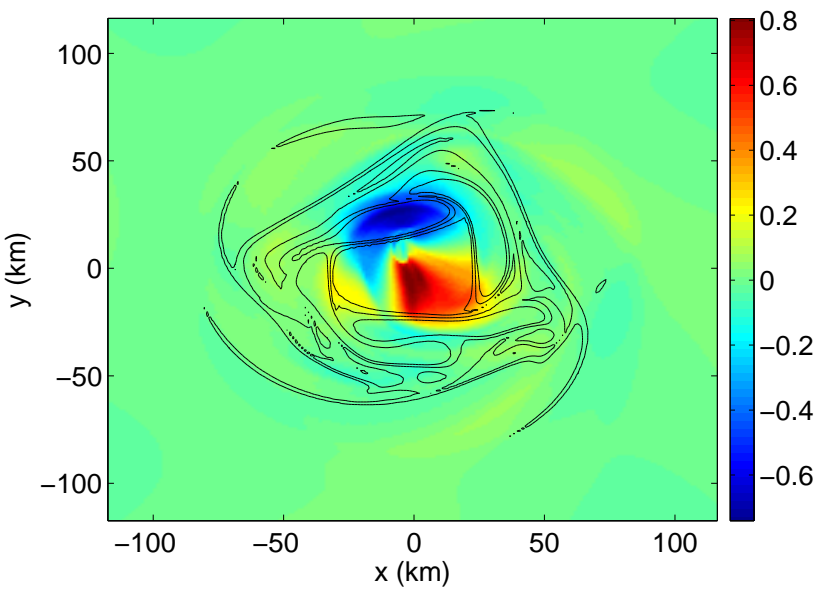

(c)

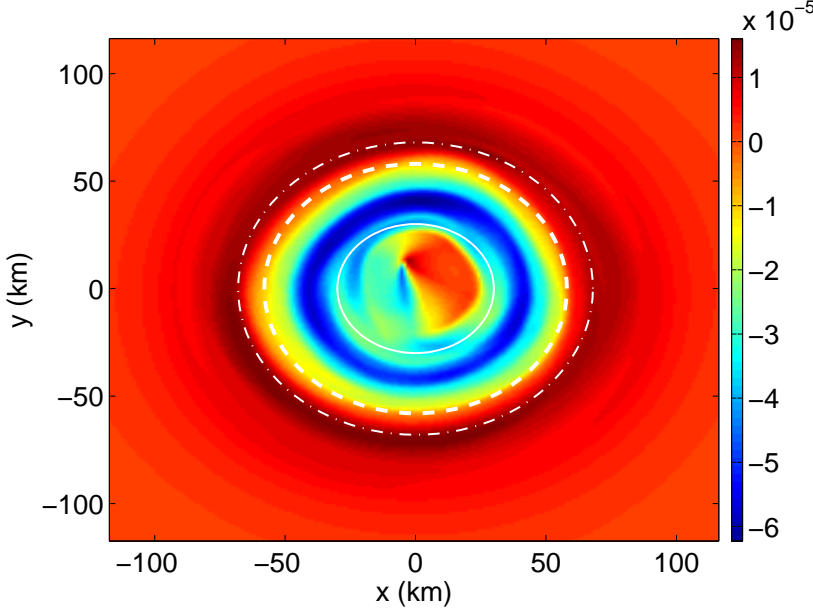

(e)

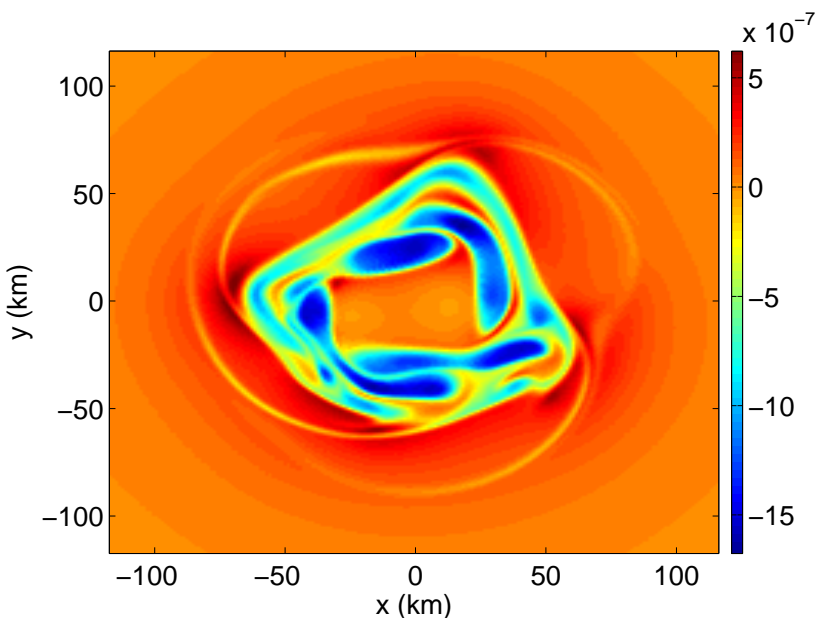

(b)

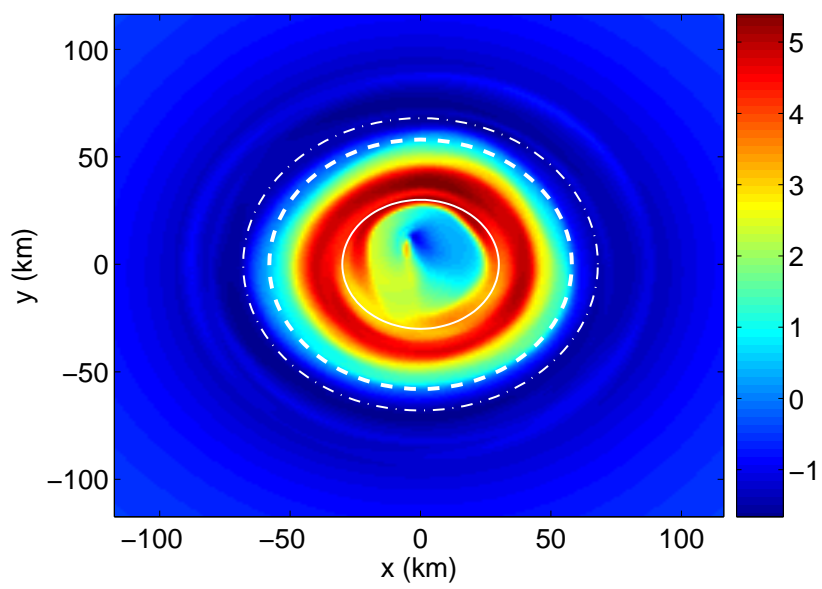

(d)

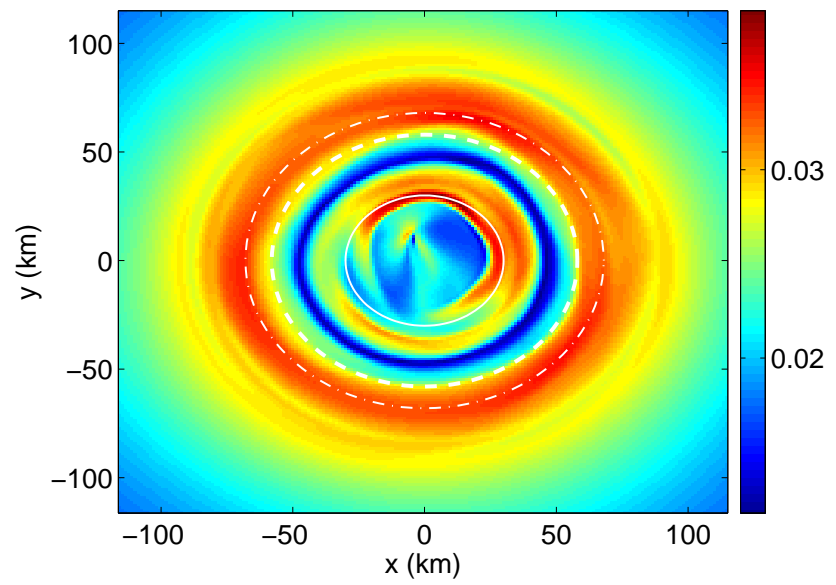

(f)

Fig. 10. (a) Relative vorticity field, (b) $Q$-field, (c) $R$-field with vorticity contours overlayed to relate structures, (d) $S$-field, (e) integrated $Q$-field $\widehat{Q}$, and (f) FTLE-field for initial time of $t_{0}=10 \mathrm{~h}$ with integration time $T=1 \mathrm{~h}$. The white contours in (d)-(f) mark the radius of maximum $\frac{\partial \omega}{\partial r}$ (inner, solid), the radius of maximum tangential wind (middle, dashed), and the radius of maximum counterpropogating $\frac{\partial \omega}{\partial r}$ (outer, dash-dot). 


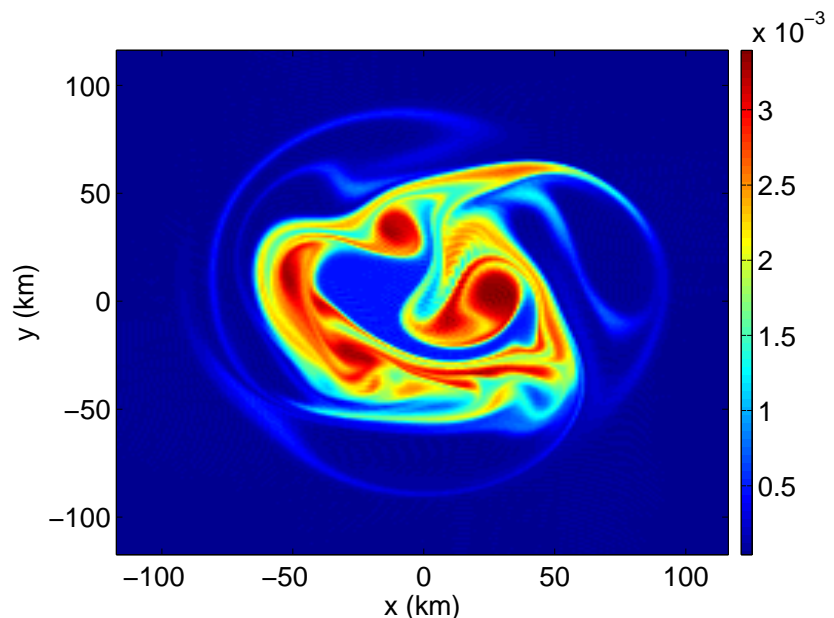

(a)

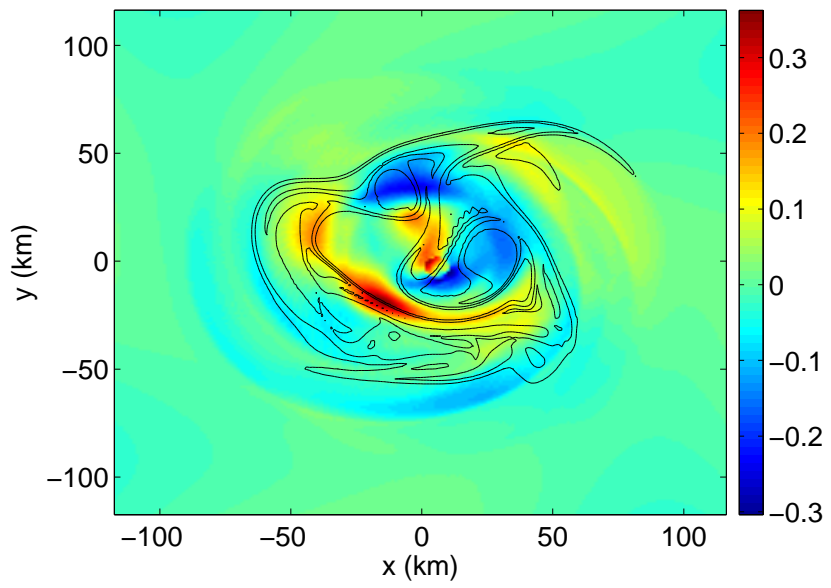

(c)

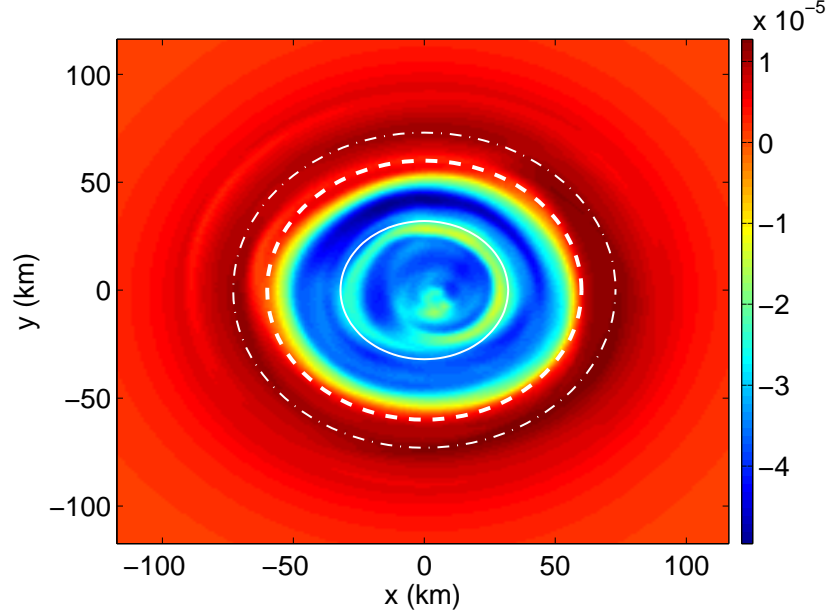

(e)

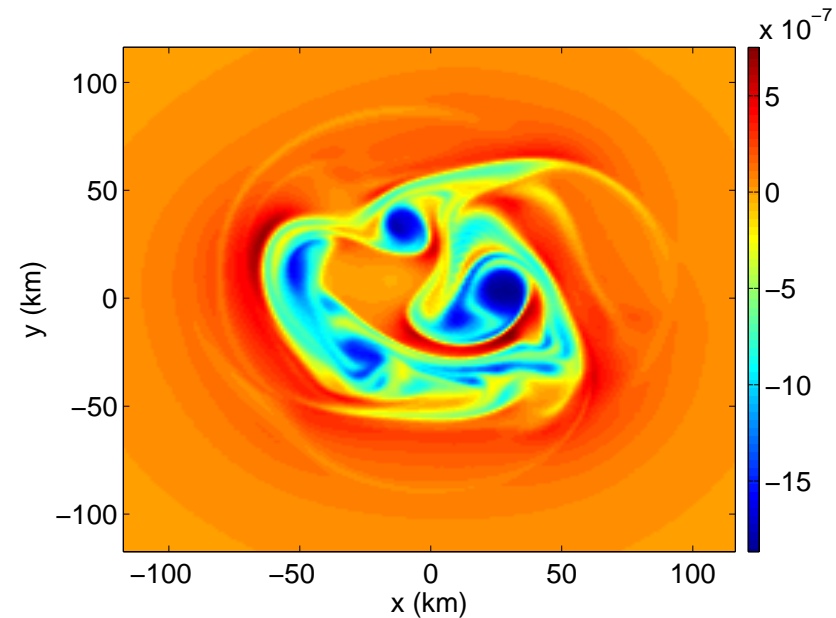

(b)

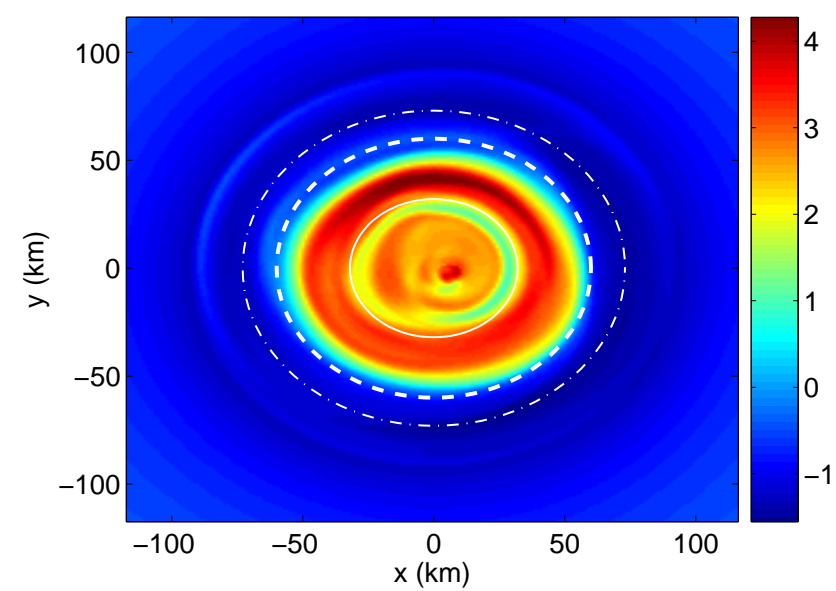

(d)

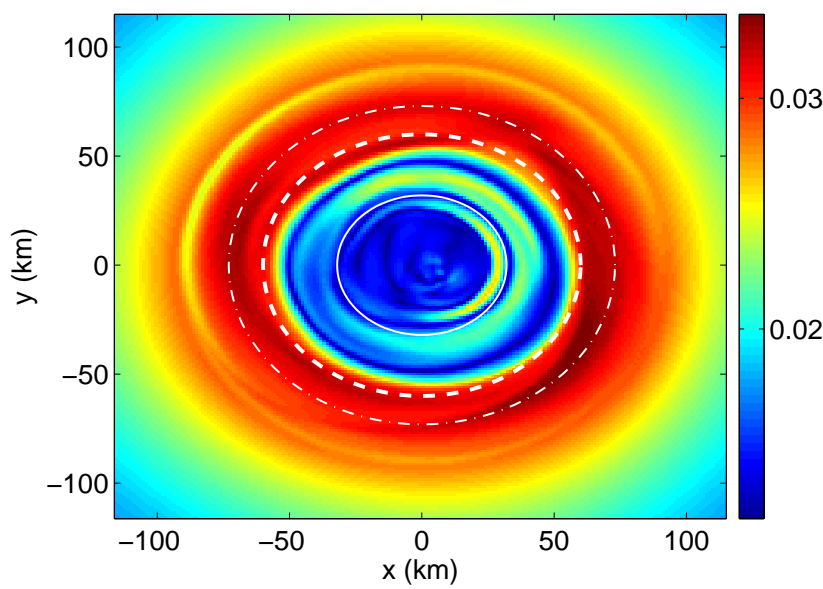

(f)

Fig. 11. (a) Relative vorticity field, (b) $Q$-field, (c) $R$-field with vorticity contours overlayed to relate structures, (d) $S$-field, (e) integrated $Q$-field $\widehat{Q}$, and (f) FTLE-field for initial time of $t_{0}=12 \mathrm{~h}$ with integration time $T=1 \mathrm{~h}$. The white contours in (d)-(f) mark the radius of maximum $\frac{\partial \omega}{\partial r}$ (inner, solid), the radius of maximum tangential wind (middle, dashed), and the radius of maximum counterpropogating $\frac{\partial \omega}{\partial r}$ (outer, dash-dot). 


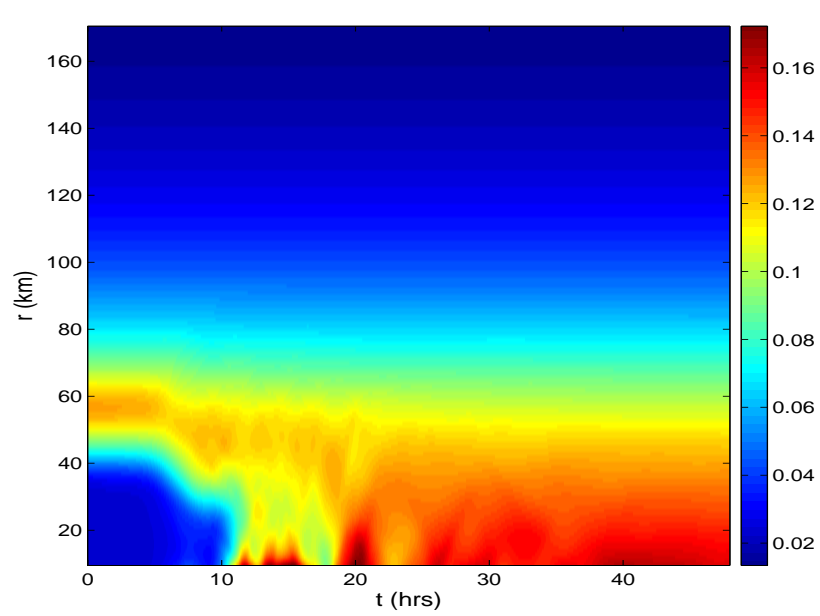

(a)

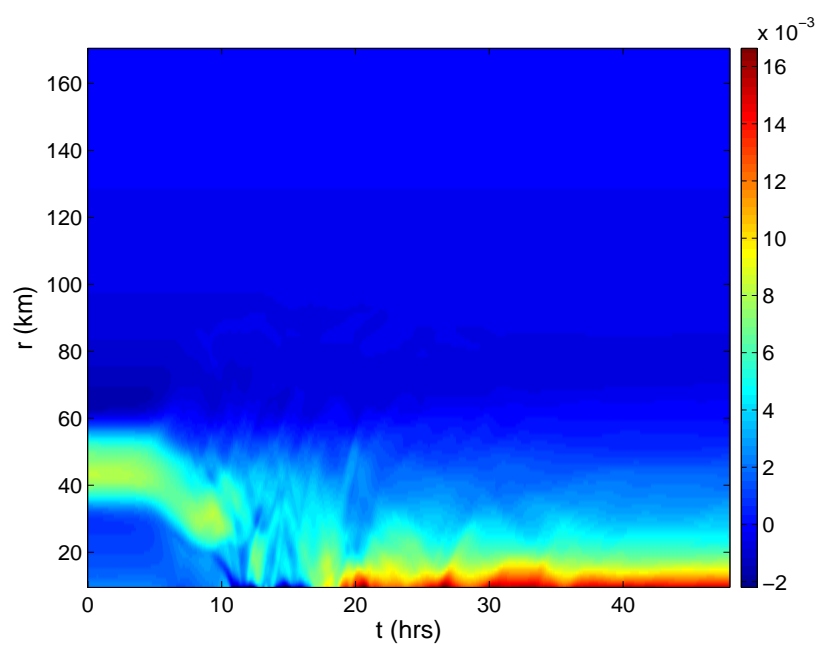

(b)

Fig. 12. (a) Averaged angular velocity $\bar{\omega}(r, t)$, and (b) averaged radial derivative $\overline{\partial \omega / \partial r}$ for $t=0-48 \mathrm{~h}$.

rates which quantify transport across shear-lines (level contours $S_{C}$ of $S$, see Sect. 3.2.3).

\subsection{Measured mixing rate}

Given a level-contour $S_{C}$ of the $S$-field and an integration time $T$, we define the mixing rate $R_{m}\left(C, t_{0}\right)$ as the area of initial conditions whose trajectories cross $S_{C}$ during $\left[t_{0}, t_{0}+T\right]$, divided by the length of the contour. This mixing rate is computed ("measured") by seeding a grid of initial conditions and counting trajectories which cross $S_{C}$.

\subsection{Predicted mixing rate}

We define a predicted mixing rate through the $R$-field as follows. Let $r_{C}$ be the average radius along $S_{C}$. If $R\left(\mathbf{x}_{0}, t_{0}\right)>0$ and $\mathbf{x}_{0}=r_{0}\left(\cos \theta_{0}, \sin \theta_{0}\right)^{*}$ with $r_{0}<r_{C}, \mathbf{x}_{0}$ is inside the circle

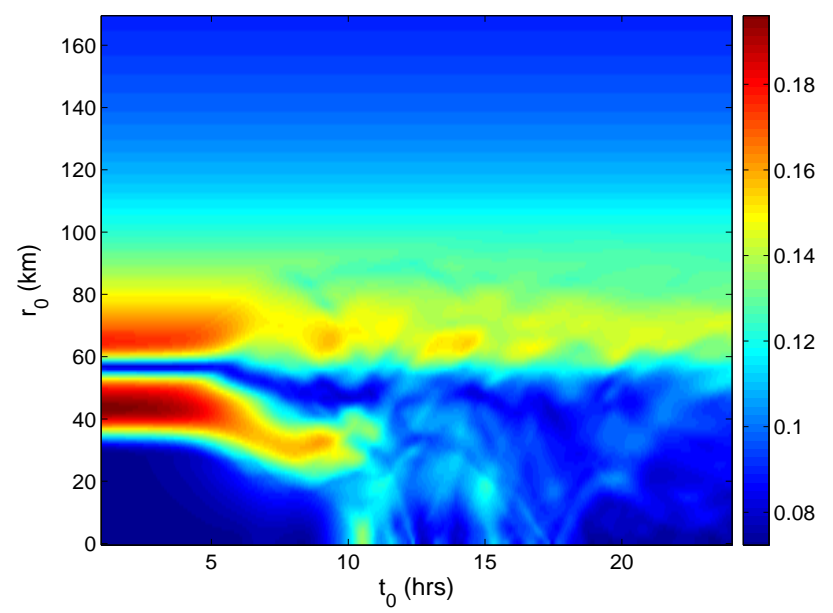

(a)

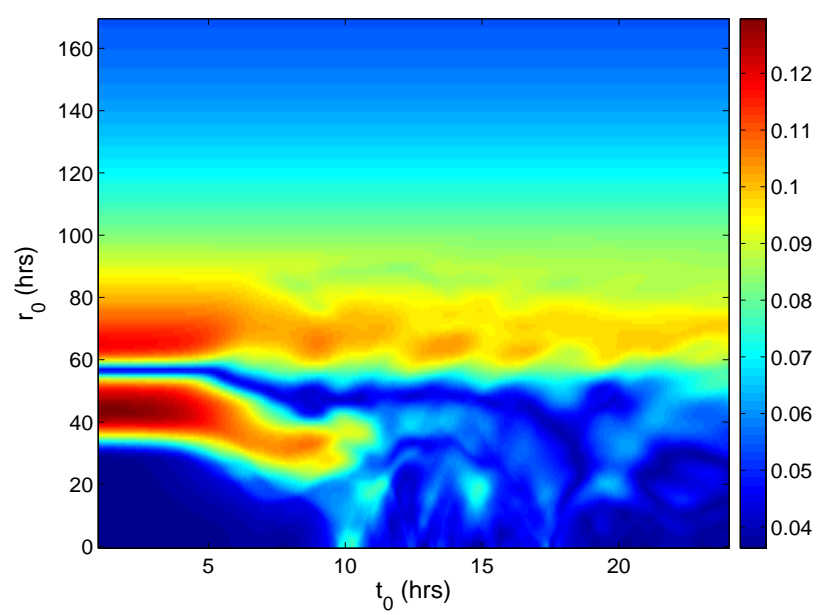

(b)

Fig. 13. Radial FTLE-values $\bar{\sigma}\left(r_{0}, t_{0}\right)$ for integration times (a) $30 \mathrm{~min}$ and (b) $1 \mathrm{~h}$.

with radius $r_{C}$, and the trajectory $\phi_{t_{0}}^{t}\left(\mathbf{x}_{0}\right)$ is repelling. Thus trajectories seeded on the ray with angle $\theta_{0}$ and radial values slightly above $r_{0}$ move outwards, towards the circle with radius $r_{C}$. This suggests to define a boundary point $\left(r_{C}-\delta\left(\theta_{0}\right), \theta_{0}\right)$ through the condition

$e^{R\left(r_{0}, \theta_{0}, t_{0}\right)} \delta\left(\theta_{0}\right)+r_{0}=r_{C}$.

Points on the $\theta_{0}$-ray above this boundary point and below $r_{C}$ can be expected to cross the $r_{C}$-circle. If $r_{0}>r_{C}$ and $R\left(\mathbf{x}_{0}, t_{0}\right)>0$, trajectories on the ray $\theta_{0}$ with radial values slightly below $r_{0}$ move inwards, towards the circle with radius $r_{C}$ again, which leads to the same boundary point (Eq. 14), now with $\delta\left(\theta_{0}\right)<0$. If $R\left(\mathbf{x}_{0}, t_{0}\right)<0$, the trajectory is attracting, and initial conditions on the opposite side of the $r_{C}$-circle move towards this circle, provided $\mathbf{x}_{0}$ is sufficiently 


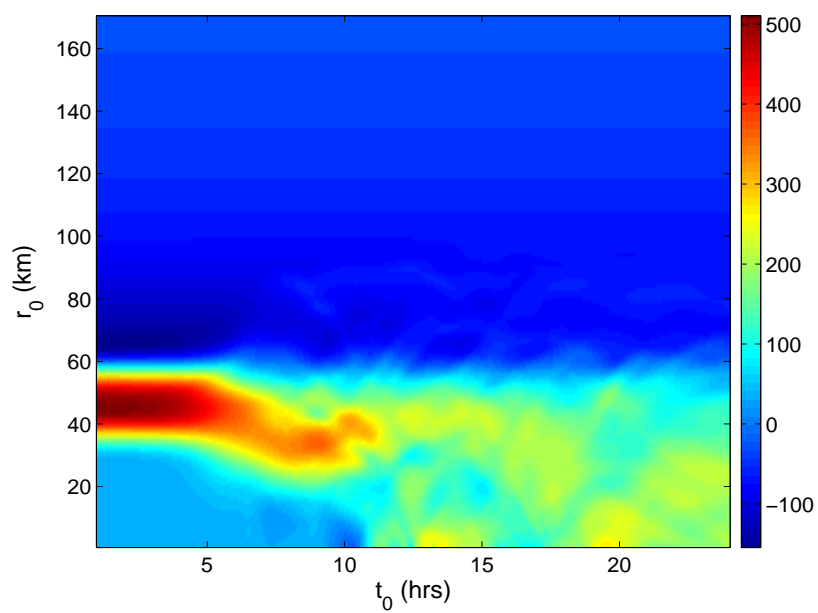

(a)

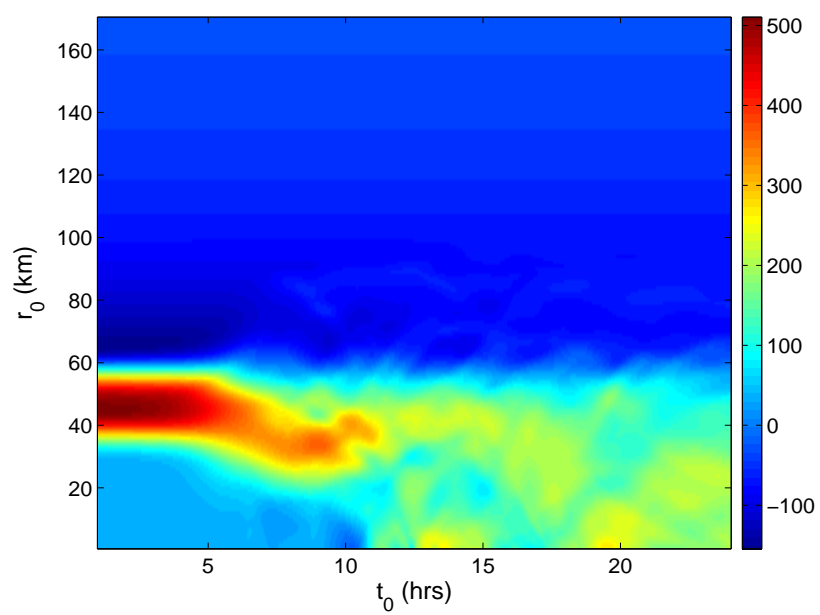

(b)

Fig. 14. Radial $S$-values $\bar{S}\left(r_{0}, t_{0}\right)$ for integration times (a) $30 \mathrm{~min}$ and (b) $1 \mathrm{hr}$.

close to that circle. The corresponding boundary point is then defined by $\left(r_{C}+\delta\left(\theta_{0}\right), \theta_{0}\right)$, where $\delta$ satisfies

$e^{R\left(r_{C}, \theta_{0}, t_{0}\right)} \delta\left(\theta_{0}\right)+r_{C}=r_{0}$.

By varying $\theta_{0}$, the conditions (Eq. 14) for $R>0$ and Eq. (15) for $R<0$ define an annulus of initial conditions around the $r_{C}$-circle, whose area we use to define the predicted mixing rate $R_{p}\left(C, t_{0}\right)$. This mixing rate is an approximation of the measured mixing rate. Of course, several approximations and simplifying assumptions are involved in this definition, but the results obtained make sense and the structure of $R_{p}$ is similar to the structure of $R_{m}$.

Color-coded plots of the mixing rates $\bar{\sigma}\left(r_{0}, t_{0}\right), \bar{S}\left(r_{0}, t_{0}\right)$, $\bar{R}\left(r_{0}, t_{0}\right), R_{m}\left(C, t_{0}\right)$, and $R_{p}\left(C, t_{0}\right)$ are displayed in Figs. 13, $14,15,16$, and 17 , respectively, for integration times $T=30 \mathrm{~min}$ and $T=1 \mathrm{~h}$. The rates $\bar{\sigma}$ and $\bar{S}$ should be compared to the average angular velocity $\bar{\omega}$ and its radial derivative

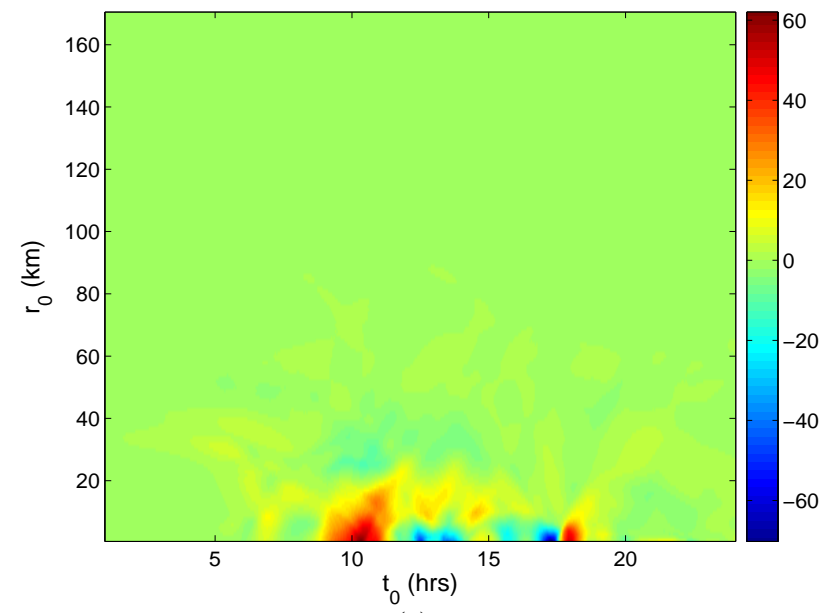

(a)

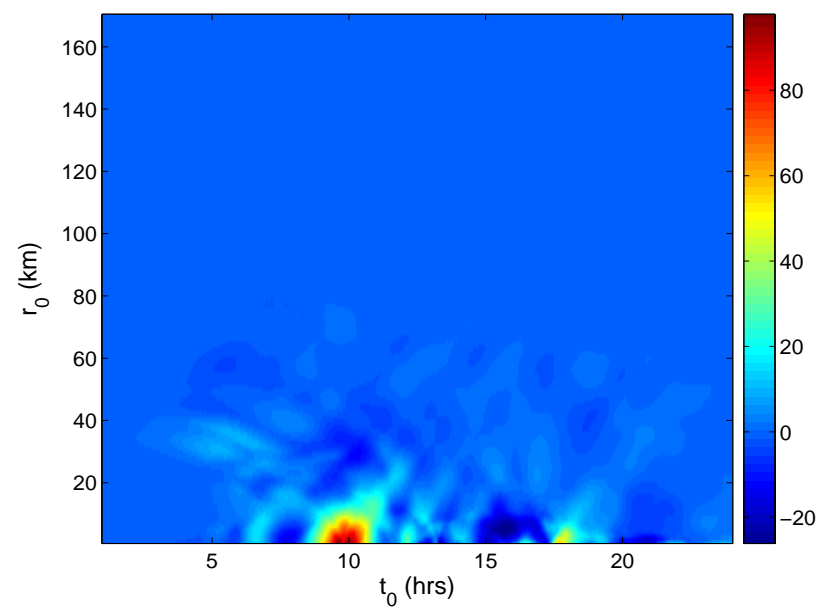

(b)

Fig. 15. Radial $R$-values $\bar{R}\left(r_{0}, t_{0}\right)$ for integration times (a) $30 \mathrm{~min}$ and (b) $1 \mathrm{~h}$.

$\overline{\partial \omega / \partial r}$ shown in Fig. 12. These rates are measures of shear and show the highest shear during the initial $6 \mathrm{~h}$, with the amount of shear dissipating as hyperbolic mixing begins to occur during the polygonal eyewall stage.

The hyperbolic mixing is captured by $\bar{R}\left(r_{0}, t_{0}\right), R_{m}\left(C, t_{0}\right)$, and $R_{p}\left(C, t_{0}\right)$. All of these rates show that high hyperbolic mixing begins with the polygonal eyewall formation at $6 \mathrm{~h}$ and continues through the transition to a high vorticity eye at $24 \mathrm{~h}$. In particular, the measured and predicted mixing rates $R_{m}\left(C, t_{0}\right)$ and $R_{p}\left(C, t_{0}\right)$ are very similar in structure, and reveal strong mixing near the zero $S$-contour $S_{0}$ (jet).

We note that Hendricks and Schubert (2009) studied mixing for the same barotropic model using the concept of effective diffusivity (Shuckburgh and Haynes, 2003; Nakamura, 1996). This quantity was computed at the initial time $t_{0}=0$, and with an integration time $t \equiv T$ (flow map $\phi_{0}^{t}$ ) varying over the full duration of the model run of $48 \mathrm{~h}$. The effective 


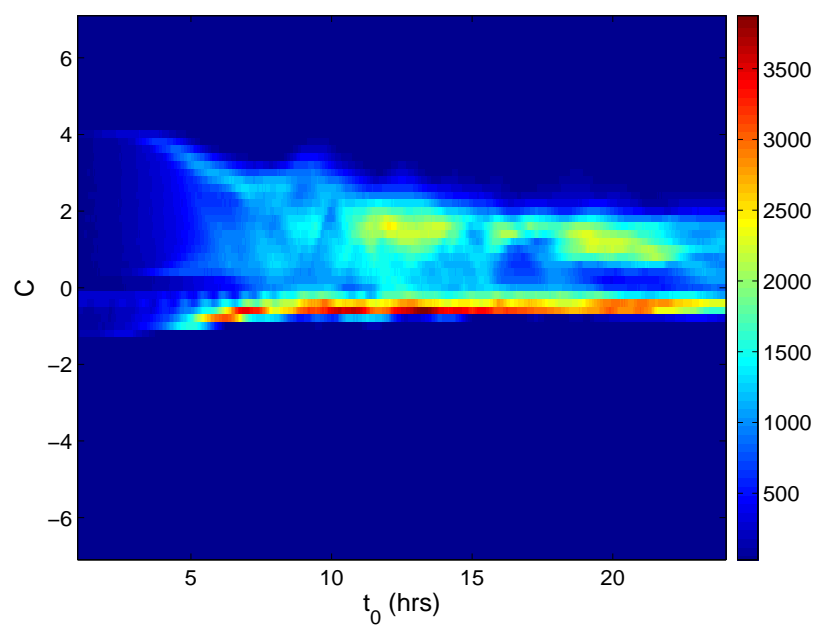

(a)

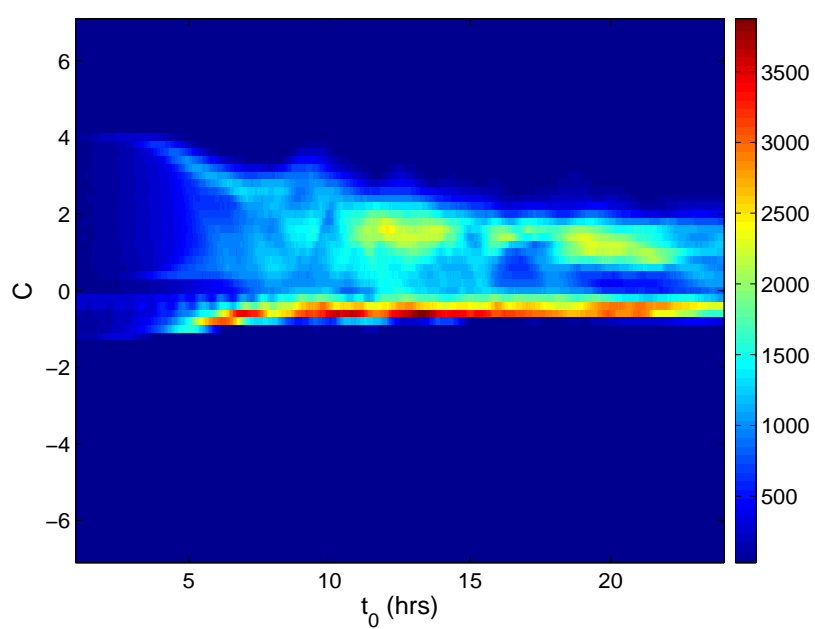

(b)

Fig. 16. Measured mixing rate versus $R_{m}\left(C, t_{0}\right)$ across shear-lines $S_{C}$ for integration times (a) $30 \mathrm{~min}$ and (b) $1 \mathrm{~h}$.

diffusivity yields a mixing rate that depends on $r$ and $t$. Our mixing rates have a similar dependence on $t_{0}$ as the mixing rate of Hendricks and Schubert (2009) had on $t$, due to our moving frame approach. The mixing rate of Hendricks and Schubert (2009) shows two radial regions, at approximately $r=30 \mathrm{~km}$ and $r=50 \mathrm{~km}$, where high mixing occurs during $t=6$ and $t=32 \mathrm{~h}$. The similar time and spatial regions associated with the same high mixing in our study occur for $\bar{\sigma}$ and $\bar{S}$ during $t_{0}=6$ and $t_{0}=24 \mathrm{~h}$, when the band of low FTLE-values (the jet) is less prominent and more hyperbolic mixing occurs. The mixing rates $R_{m}\left(C, t_{0}\right)$ and $R_{p}\left(C, t_{0}\right)$ show very similar times of high mixing as the mixing rate of Hendricks and Schubert (2009), and the $S$-contours for which these high rates occur are located approximately at the same radial values noted above.

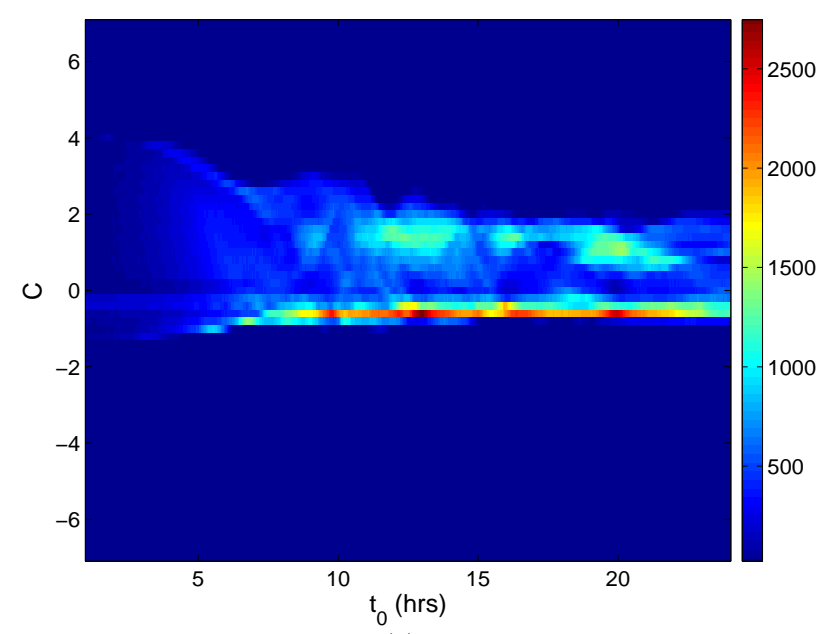

(a)

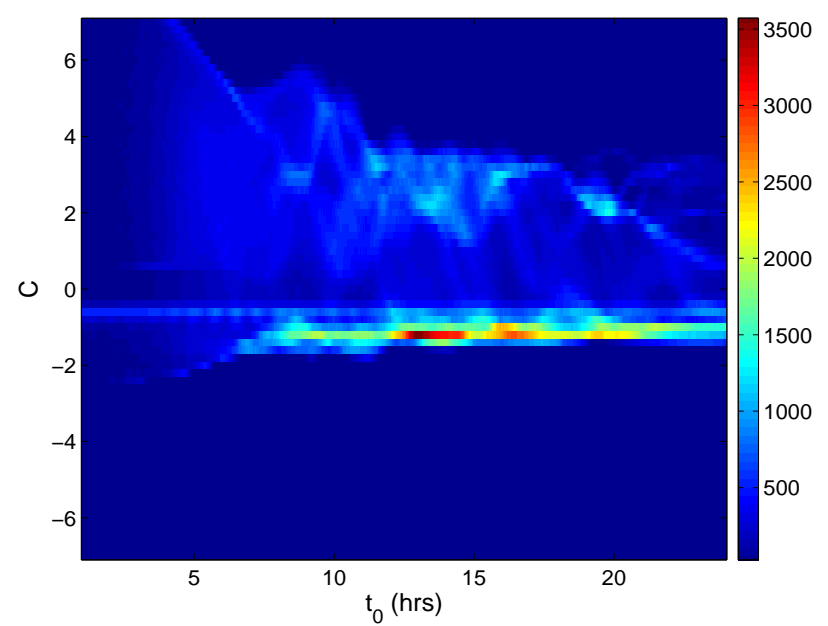

(b)

Fig. 17. Predicted mixing rate $R_{p}\left(C, t_{0}\right)$ across shear-lines $S_{C}$ for integration times (a) $30 \mathrm{~min}$ and (b) $1 \mathrm{~h}$.

High $R$-values determine sets of trajectories that show growth orthogonal to the Lagrangian velocity, and result in filamentation that enables turbulent diffusion to occur. The $R$-field gives an advective measure that converges on very short time scales, yet still yields similar mixing rates as the effective diffusivity obtained by integration over the full model time.

\section{Conclusions}

We have characterized Lagrangian mixing in a twodimensional, nondivergent barotropic model for hurricanelike vortices through several diagnostic techniques. For this model, the field of finite-time Lyapunov exponents provided a measure of total particle separation, but it did not separate the effects of hyperbolicity and shear, and did not show 
distinct coherent structures. In order to separate the effects of the high shearing occurring in the model from hyperbolic mixing, the trajectory separation was decomposed in directions along and normal to the Lagrangian velocity. This decomposition gave rise to two Lagrangian fields, the $R$-field and the $S$-field, which quantified the relative contributions of hyperbolicity and shear to the mixing process, respectively. In this approach, shear-lines and shear-strengths were identified with level-contours and level-values of the $S$-field.

The $R$-field showed coherent structures which impacted the mixing and mesovortex interaction, even through high shear. The outer ridges of the $R$-field were also involved in protecting the mesovortices from environmental flow, and the persistance of the ridges was associated with the dominance of particular mesovortices over others. In contrast to the other Lagrangian methods showed that the $R$-field was able to distinguish not only regions of high mixing, but also the structures that were involved in the evolution and annihilation of mesovortices. Future work will be devoted to study the impact of coherent structures on mesovortex interaction during tropical cyclogenesis and its role for the evolution of a wave critical layer.

The impact of the coherent structures on the mixing process was quantified in terms of time- and space-dependent mixing rates, with the spatial dependence displayed as a function of shear-strength as well as a function of the (average) radius of shear-lines. Overall, the moving-frame approach used in this paper provides time-dependent mixing rates that isolate mixing events occurring in particular time windows.

The methods used here led to mixing profiles of similar structure as in Hendricks and Schubert (2009), but with a moving time frame and with fast convergence in Lagrangian fields integrated over short times. The $S$-field provided a natural choice of contours for varying initial time that allowed to quantify mixing by determining transport across them.

Future work will address the impact of hyperbolicity in the presence of high shear in a three-dimensional setting, where planar shear and movement orthogonal to the shear are separated using suitable extensions of the fields introduced in this paper. These techniques will be applied to a realistic, three-dimensional hurricane model that has both shearing and hyperbolic components governing the mixing processes. The focus of this work will be rather different from that of a recent paper by Sapsis and Haller (2009), who studied mixing in a three-dimensional hurricane model by computing Lagrangian quantities along a slow manifold.
Acknowledgements. This work was supported by the National Science Foundation under NSF Cooperative Agreement ATM0715426. The authors thank Eric Hendricks, Arthur Jamshidi, Kate Musgrave, Christopher Rozoff, Jonathan Vigh, and Michael Riemer for their discussions. The authors would also like to thank the anonymous referee and Tim Dunkerton for their helpful comments and criticisms.

Edited by: P. Haynes

\section{References}

Andrews, D. G. and McIntyre, M. E.: An exact theory of nonlinear waves on a Lagrangian mean flow, J. Fluid Mech., 89, 609-646, 1978.

Braun, S. A., Montgomery, M. T., and Pu, Z.: High-resolution simulation of Hurricane Bonnie (1998), Part I: The organization of eyewall vertical motion. J. Atmos. Sci., 63, 19-42, 2006.

Bryan, G. H. and Rotunno, R.: The maximum intensity of tropical cyclones in axisymmetric numerical model simulations. Mon. Weather Rev., 137, 1170-1789, 2009a.

Bryan, G. H. and Rotunno, R.: Evaluation of an analytical model for the maximum intensity of tropical cyclones, J. Atmos. Sci., 66, 3042-3060, 2009b.

Cram, T. A., Persing, J., Montgomery, M. T., and Braun, S. A.: A Lagrangian trajectory view on mixing between the eye, eyewall, and environment using a high resolution simulation of Hurricane Bonnie (1998), J. Atmos. Sci., 64, 1835-1856, 2007.

Dunkerton, T.: A Lagrangian mean theory of wave, mean-flow interaction with applications to nonacceleration and its breakdown, Rev. Geophys., 18(2), 387-400, 1980.

Dunkerton, T. J., Montgomery, M. T., and Wang, Z.: Tropical cyclogenesis in a tropical wave critical layer: easterly waves, Atmos. Chem. Phys., 9, 5587-5646, 2009, http://www.atmos-chem-phys.net/9/5587/2009/.

Frank, W. M. and Ritchie, E. A.: Effects of environmental flow upon tropical cyclone structure, Mon. Weather Rev., 127, 2044-2061, 1999.

Frank, W. M. and Ritchie, E. A.: Effects of vertical wind shear on the intensity and structure of numerically simulated hurricanes, Mon. Weather Rev., 129, 2249-2269, 2001.

Green, M. A., Rowley, C. W., and Haller, G.: Detection of Lagrangian coherent structures in 3D turbulence, J. Fluid Mech., 572, 111-120, 2007.

Haller, G.: Finding finite-time invariant manifolds in twodimensioanl velocity fields, Chaos, 10, 99-108, 2000.

Haller, G.: Lagrangian structures and the rate of strain in a partition of two-dimensional turbulence, Physics of Fluids, 13, 33653385, 2001.

Haller, G.: Lagrangian coherent structures from approximate velocity data, Phys. Fluids, 14, 1851-1861, 2002.

Haller, G. and Iacono, R.: Stretching, alignment, and shear in slowly varying velocity fields, Phys. Rev. E, 68, 056034 1-6, 2003.

Haller, G. and Poje, A. C.: Finite time transport in aperiodic flows, Physica D, 119, 352-380, 1997.

Haller, G. and Yuan, G.: Lagrangian coherent structures and mixing in two-dimensional turbulence, Physica D, 147, 352-370, 2000. 
Hendricks, E. A. and Schubert, W. H.: Transport and mixing in idealized barotropic hurricane-like vortices, Q. J. Roy. Meteorol. Soc, 135(643), 1456-1470, 2009.

Huber, M., McWilliams, J. C., and Ghil, M.: A climatology of turbulent dispersion in the troposphere, J. Atmos. Sci., 58, 23772394, 2001.

Ide, K., Small, D., and Wiggins, S.: Distinguished hyperbolic trajectories in time-dependent fluid flows: Analytical and computational approach for velocity fields defined as data sets, Nonlinear Proc. Geoph., 9, 237-263, 2002.

Joseph, B. and Legras, B.: Relation between kinematic boundaries, stirring, and barriers for the antarctic polar vortex, J. Atmos. Sci., 59, 1198-1212, 2001.

Koh, T. Y. and Legras, B.: Hyperbolic lines and the stratospheric polar vortex, Chaos, 12(2), 382-394, 2002.

Kossin, J. P. and Eastin, M. D.: Two distinct regimes in the kinematic and thermodynamic structure of the hurricane eye and eyewall, J. Atmos. Sci., 58, 1079-1090, 2001.

Kossin, J. P. and Schubert, W. H.: Mesovortices, polygonal flow patterns, and rapid pressure falls in hurricane-like vortices, J. Atmos. Sci., 58, 2196-2209, 2001.

McIntyre, M. E.: Toward a Lagrangian-mean description of stratospheric circulations and chemical transports, Philos. T. R. Soc. Lond., 296, 129-148, 1980.

Montgomery, M. T. and $\mathrm{Lu}, \mathrm{C}$. : Free waves on barotropic vortices, Part I: Eigenmode structure, J. Atmos. Sci., 54, 1868-1885, 1997.

Montgomery, M. T., Vladimirov, V. A., and Denissenko, P. D.: An experimental study on hurricane mesovortices, J. Fluid Mech., 471, 1-32, 2002.

Montgomery, M. T., Bell, M. M., Aberson, S. D., and Black, M. L.: Hurricane Isabel (2003): New insights into the physics of intense storms, Part I: Mean vortex structure and maximum intensity estimates, B. Am. Meteorol. Soc., 87, 1349-1354, 2006.
Nakamura, N.: Two-dimensional mixing, edge formation, and permeability diagnosed in area coordinates, J. Atmos. Sci., 53, 1524-1537, 1996.

Ottino, J. M.: The Kinematics of Mixing: Stretching, Chaos, and Transport, Cambridge University Press, 1989.

Rotunno, R. and Emanuel, K.: An air-sea interaction theory for tropical cyclones, Part II: Evolutionary study using a nonhydrostatic axisymmetric numerical model, J. Atmos. Sci., 44, 542$561,1987$.

Rutherford, B., Dangelmayr, G., Persing, J., Kirby, M., and Montgomery, M.T.: Lagrangian mixing in an axisymmetric hurricane model, Atmos. Chem. Phys. Discuss., 9, 18545-18596, 2009, http://www.atmos-chem-phys-discuss.net/9/18545/2009/.

Sapsis, T. and Haller, G.: Inertial particle dynamics in a hurricane, J. Atmos. Sci., 66, 2481-2492, 2009.

Schubert, W. H., Montgomery, M. T., Taft, R. K., Guinn, T. A., Fulton, S. R., Kossin, J. P., and Edwards, J. P.: Polygonal eyewalls, asymmetric eye contraction, and potential vorticity mixing in hurricanes, J. Atmos. Sci., 56, 1197-1223, 1999.

Shadden, S. C., Lekein, F., and Marsden, J. E.: Definition and properties of Lagrangian coherent structures from finite-time Lyapunov exponents in two-dimensional aperiodic flows, Physica D, 212, 271-304, 2005.

Shuckburgh, E. and Haynes, P.: Diagnosing transport and mixing using a tracer-based coordinate system, Phys. Fluids, 15, 33423357, 2003.

Willoughby, H. E.: Tropical cyclone eye thermodynamics, Mon. Weather Rev., 126, 3053-3067, 2001.

Wirth, V. and Dunkerton, T. J.: A unified perspective on the dynamics of axisymmetric hurricanes and monsoons, J. Atmos. Sci., 63, 2529-2547, 2006. 